\title{
HW/SW co-design of a visual SLAM application
}

\author{
Jonathan Piat ${ }^{1,2} \cdot$ Philippe Fillatreau $^{4} \cdot$ Daniel Tortei $^{1,2} \cdot$ Francois Brenot $^{1,3} \cdot$ Michel Devy $^{1}$
}

\begin{abstract}
Vision-based advanced driver assistance systems (ADAS), appeared in the 2000s, are increasingly integrated on-board mass-produced vehicles, as off-the-shelf low-cost cameras are now available. But ADAS implement most of the timespecific and basic functionalities such as lane departure or control of the distance to other vehicles. Integrating accurate localization and mapping functionalities meeting the constraints of ADAS (high-throughput, low-consumption, and smalldesign footprint) would pave the way towards obstacle detection, identification and tracking on-board vehicles at potential high speed. While the SLAM problem has been widely addressed by the robotics community, very few embedded operational implementations can be found, and they do not meet the ADAS-related constraints. In this paper, we implement the first 3D monocular EKF-SLAM chain on a heterogeneous architecture, on a single System on Chip (SoC), meeting these constraints. In order to do so, we picked up a standard co-design method (Shaout et al. Specification and modeling of hw/sw co-design for heterogeneous embedded systems, 2009) and adapted it to the implementation of potentially any of such complex processing chains. The refined method encompasses a hardware-in-the-loop approach allowing to progressively integrate hardware accelerators on the basis of a systematic rule. We also have designed original hardware accelerators for all the image processing functions involved, and for some algebraic operations involved in the filtering process.
\end{abstract}

Keywords FPGA $\cdot$ Co-design $\cdot$ SLAM $\cdot$ Machine-vision $\cdot$ ADAS

Jonathan Piat

piat.jonathan@gmail.com

Philippe Fillatreau

philippe.fillatreau@enit.fr

Daniel Tortei

dtertei@laas.fr

Francois Brenot

fbrenot@laas.fr

Michel Devy

michel.devy@laas.fr

1 CNRS, LAAS, 7 avenue du colonel Roche, 31400 Toulouse, France

2 Univ de Toulouse, UPS, LAAS, 31400 Toulouse, France

3 Institut National Polytechnique de Toulouse, INPT - University of Toulouse, 4 Allee Emile Monso, 31030 Toulouse, France

4 Laboratoire Genie de Production de l'Ecole Nationale d'Ingenieurs de Tarbes (LGP-ENIT), Institut National Polytechnique de Toulouse (INPT), University of Toulouse, 47 avenue d'Azereix, BP1629, 65016 Tarbes Cedex, France

\section{Introduction}

Advanced driver assistance systems (ADAS) appeared in the 2000s and are now increasingly found on-board mass-produced vehicles. They involve various sensors (cameras, lidar, others) and signal or image processing functionalities (e.g. extraction and analysis of features detected in acquired signals or images), see [33]. ADAS-related concepts have been notably validated by research on perception or navigation for autonomous mobile robots since the 1980s. Nevertheless, ADAS implement most of the time specific and independent functionalities, such as the control of distance to other vehicles, or the detection of driver hypovigilance or lane departure, see [27]. Functionalities such as accurate vehicle localization and sparse vehicle environment mapping would allow paving the way towards obstacle detection, identification and tracking (notably thanks to the reduction of the complexity of the associated data processing) and towards autonomous navigation of road vehicles at high speed.

Self-localization and environment modelling have been central issues for research on mobile robots since the $1980 \mathrm{~s}$ [10], although the SLAM acronym appeared towards the end 
of the 1990s [14]. Various sensors have been used for the associated perception tasks, e.g., ultrasonic sensors, laser range finders, and cameras. The emergence of off-the-shelf low-cost cameras allows using them in monocular, stereovision, or panoramic vision-based ADAS systems, see [18].

The simultaneous localization and mapping (SLAM) issue has now been widely explored by the robotics community, and numerous academic solutions have been proposed. However, very few complete embedded operational chains meeting the constraints associated with ADAS (fast processing times, low-consumption, small-design footprint) can be found in the literature. This is partly due to the intrinsic complexity of the advanced image processing algorithms involved and to the large volume of data (number of pixels) to be processed.

The computer vision and robotics perception communities started in the late 1990s-early 2000s to study the design of specific, embedded and real-time architectures, involving programmable logics, for advanced image processing functionalities, see [35]. Much progress remains to be done about vision-based SLAM, a complex image processing and numerical filtering chain. To embed such a complex functionality on an ADAS, one must explore the possibilities offered by available execution resources, to take advantage of their specific properties. This leads the designer to formulate a heterogeneous (or not) processing architecture involving both general purpose processing units and application-specific hardware accelerators. To define an efficient distribution of the computational load over these two kinds of operators, the design needs to be undertaken following joint design (called co-design) approaches.

This paper deals with the definition of a heterogeneous architecture for the integration of a complete monocular 3D extended Kalman filter (EKF) vision-based SLAM processing chain meeting the constraints of an ADAS. To achieve that, we have studied the introduction of a suitable co-design methodology associated with a hardware-in-the-loop (HIL) approach allowing to progressively integrate specifically designed hardware accelerators.

The contribution is threefold :

- we have selected in the literature a standard and generic co-design approach [38] which we have (1) refined to allow the integration of a complex processing chain such as a vision-based monocular SLAM and (2) adapted to encompass a hardware-in-the-loop approach allowing to progressively integrate hardware accelerators; in our approach, the hardware accelerators are integrated one by one, based on the use of a systematic rule based on the results of the profiling of the heterogeneous SLAM chain under construction. The refinement and adaptation brought to the state-of-the-art method we picked up [38] are not specific to the nature of the processing chain

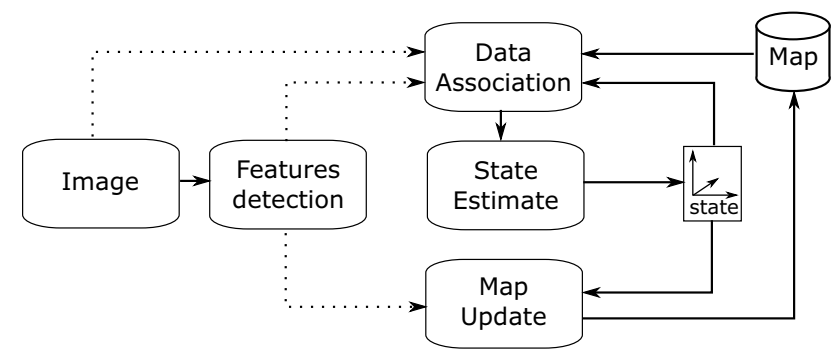

Fig. 1 Simultaneous localization and mapping-SLAM

developed here, and can be reused for any advanced signal or image processing chain.

- we have used this refined co-design method to integrate the first complete monocular 3D EKF-SLAM chain on a heterogeneous (hardware/software) architecture on a single SoC. The obtained performances meet the constraints (processing times, power consumption, design footprint) of the ADAS.

- we have developed and validated original hardware accelerators (reusable for any embedded SLAM application or vision application involving the same functionalities) of all the image processing and of some data processing functions involved in the numerical filtering steps (Fig. 1).

This work was conducted in the context of the Development of an Integrated Camera for Transport Applications (DICTA) cooperative project. The goal of this project is to integrate advanced ADAS functionalities on the iCam smart camera developed by the Delta Technologies company, see [13].

This paper is organized as follows. In Sect. 2, we present a state-of-the-art spanning over the basic SLAM principles and different approaches found in the literature, the basics of extended Kalman filtering, the operational vision-based SLAM implementations in the community and at the LAAS laboratory, and the hardware/software co-design methodologies; this section ends by a synthesis of the working hypothesis, the scientific approach followed and the main contributions of this work. Section 3 presents the refined and adapted generic co-design methodology proposed here, and details the successive steps of the development of our heterogeneous monocular 3D EKF-SLAM chain. The rest of the paper is organized according to these steps. Section 4 deals with step 1 of our co-design methodology, which defines the architecture model, the application model, and the constraints on the embedded system. Section 5 presents iteration 1, performing the initial platform specification, the application model refinement, and the implementation of a software-only prototype. Section 6 deals with iterations 2-4 (iterative integration of original hardware accelerators). 
Section 7 presents the final embedded prototype, discusses its validation through the experimental results obtained and compares it to state-of-the-art implementations. Section 8 presents our global conclusions and our future works.

\section{State of the art and motivation}

\subsection{SLAM: general principle}

In the framework of the autonomous navigation of a mobile robot or vehicle, the SLAM issue deals with the problem of incrementally building a map of an unknown environment explored by the vehicle, while simultaneously keeping track of the vehicle pose (position and orientation) in this environment (see Fig. 2). Various exteroceptive sensors have been used to sense the environment (e.g. ultrasonic sensors, laser range finders or one or several cameras). Tracking and matching landmarks over time allow updating the environment map: the positions of already perceived landmarks are refined while newly perceived landmarks are integrated into the map, see [14]. Proprioceptive sensors (e.g. odometry or inertial measurement units-IMU) provide an estimate of the robot pose, notably allowing reducing the complexity of the landmarks tracking process. Tasks involved in the SLAM process may be described as front-end perception tasks, and back-end pose estimation and map update tasks, see [7, 37].

\subsection{SLAM: different approaches}

The approaches found in the literature for the SLAM process may be classified mainly into filtering-based approaches, and approaches based on bundle adjustment optimization.

Historically, filtering-based SLAM approaches (based on the use of an extended Kalman filter-EKF) have been proposed first (see for example [31]) and have remained very popular since then. Filtering-based approaches use probabilistic data to describe the robot state (robot pose) and the landmark positions in the environment map. Matching landmarks allows refining the map and the robot pose through a numerical process (the extended Kalman filter is widely used at this stage). EKF-based SLAM exhibits wellknown limitations. First, the approach is complex, as EKFbased SLAM requires to integrate all landmarks positions and the robot pose in the same state vector and to manage their uncertainties (large covariance matrix) in the filtering process. The EKF-SLAM complexity is evaluated in Sola [40] to $o\left(k \cdot n^{2}\right)$, where $n$ is the global number of landmarks in the environment map and $k$ is the number of landmarks observed at each algorithm iteration. Second, consistency problems appear, due to errors related to the linearization performed in the neighborhood of the system state, and to possible wrong landmark matches. Thus, the community

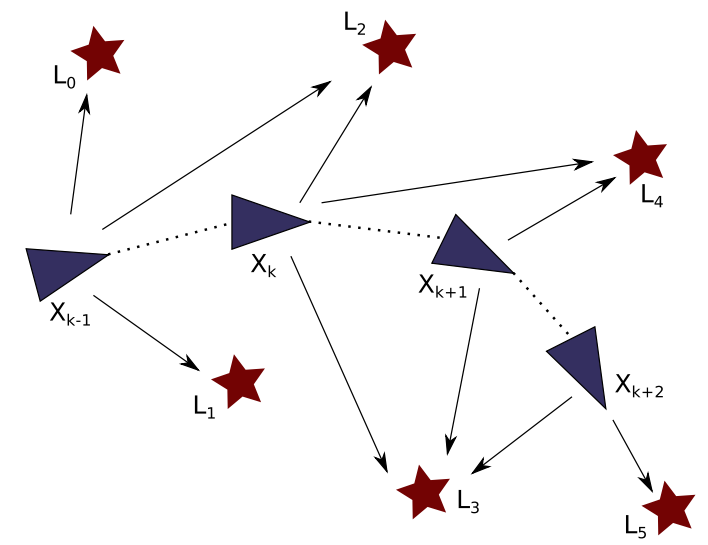

Fig. 2 Principles of the simultaneous localization and mapping problem: a mobile object (blue triangle) computes its local state $X_{k}$ using static information in the world frame (landmarks $L_{n}$ ) and previous state(s) $X_{k-n}$

has explored other solutions. In Montemerlo et al. [29], the authors propose the FastSLAM algorithm, based on the use of a particle filter instead of the EKF. On the one hand, this approach reduces complexity by decorrelating the robot pose from the landmarks positions, but on the other hand, this approach also exhibits similar complexity and consistency issues (especially as the number of particles grows with the size of the environment map).

Another approach has been getting more and more interesting over the last few years: bundle adjustment SLAM performs optimization over selected key frames in the sequence of images acquired by the robot, see [30, 42]; incoming key frames correspond (for example) to images for which too few features matches are obtained, and the uncertainty of the robot pose is too high; key frames should be spatially distributed, and may be kept in limited number using a temporally sliding window.

In Strasdat et al. [42], the authors compare filteringbased and bundle adjustment-based SLAM approaches for purely software implementations, and conclude that key frame-based optimisation provide the best accuracy per unit of computing time if powerful processing units and large amounts of interest points can be used. But, the authors show that filtering-based approaches still appear beneficial for small processing budgets (as is the case in our study).

\subsection{SLAM: extended Kalman filter}

Filtering is the process of estimating the system state from noisy, partial and/or indirect state measurements.

The Kalman filter provides an optimal state estimate in the event of noisy measurements, assuming a Gaussian distributed noise and a linear process model. The filter relies on the recursive estimation of the state and the stochastic 
properties of the process. Starting from an initial state and a stochastic model of the process and data sources, the filter predicts the future state of the system together with its prediction error. The predicted state is then compared to the actual state observed from the measurements, to update the stochastic model and refine the state estimate. The updated stochastic model will help achieve better state prediction for the next iterations of the process.

The state prediction step is formulated as follows:

$$
\begin{aligned}
\hat{\mathbf{x}}^{+} & =\mathbf{F} \hat{\mathbf{x}} \\
\mathbf{P}^{+} & =\mathbf{F P F} \mathbf{F}^{T}+\mathbf{G Q G}^{T},
\end{aligned}
$$

where $\hat{\mathbf{x}}^{+}$the predicted state, $\hat{\mathbf{x}}$ the current state, $\mathbf{F}$ the state transition matrix, $\mathbf{P}$ the state error covariance matrix, $\mathbf{P}^{+}$the a posteriori error covariance matrix, and $\mathbf{Q}$ the covariance of the process noise.

The state update step is formulated as follows:

$$
\begin{aligned}
& \mathbf{Z}=\mathbf{H} \mathbf{P} \mathbf{H}^{T}+\mathbf{R}, \\
& \mathbf{K}=\mathbf{P} \mathbf{H}^{T} \cdot \mathbf{Z}^{-1}, \\
& \hat{\mathbf{x}}^{+}=\hat{\mathbf{x}}+\mathbf{K}(\mathbf{y}-\mathbf{H} \hat{\mathbf{x}}), \\
& \hat{\mathbf{P}}^{+}=\hat{\mathbf{P}}-\mathbf{K} \mathbf{Z} \mathbf{K}^{T},
\end{aligned}
$$

where $\mathbf{H}$ is the observation model matrix, $\mathbf{Z}$ is the innovation (difference between the observation and the predicted state) covariance matrix, $\mathbf{K}$ is the Kalman gain matrix that allows updating the state and state error covariance matrix.

The extended Kalman filter extends this framework to the case of a non-linear process model. In the state prediction and state update steps, the observation and prediction functions are linearized around the current state of the system. An extended Kalman filter iteration for the visual SLAM problem can be formulated as follows:

1. The state is predicted using a movement model of the system that can integrate proprioceptive data (IMU, odometry...), or a simpler model like a constant speed model.

2. The state observation step consists in observing map elements (landmarks) as features to be observed in the images. These observations are iteratively integrated into the filter following the update process step.

3. Features are detected in the image and integrated as landmarks in the process state. These map points will later be used for the state observation step of the filter.

This process implies, on the computer-vision side, the ability to:

1. detect image features

2. describe image features in the world frame
3. accurately match image features in a frame sequence

These tasks must be performed with as much accuracy as possible since wrong image features or bad matches may endanger the filter stability.

\subsection{Operational vision-based embedded SLAM implementations}

There are still very few real-time embedded operational full SLAM processing chains in the literature. Early works have proposed systems based on the use of telemeters or infrared (IR) sensors. For example, Abrate et al. [1] have proposed a solution involving eight low-cost IR sensors. The SLAM processing chain was implemented on a low-cost CPU Motorola 68331 processor. The processing times obtained limited the robot speed to $0.6 \mathrm{~m} / \mathrm{s}$. The emergence of lowcost cameras and the improvements of embedded processors performances have allowed the implementation of embedded vision-based SLAM solutions in the last few years. Bonato et al. [5] have proposed a system based on the use of four cameras (resolution: $320 \times 240$ pixels). The authors have implemented a SIFT interest points descriptor on a Stratix ${ }^{\circledR}$ II FPGA. The hardware blocks are coded in Handel-C and the communication interfaces and cameras interfaces are coded in VHDL. The authors foresee performances for a complete vision-based 2D EKF-SLAM chain of $14 \mathrm{~Hz}$ with a consumption of $1.3 \mathrm{~W}$. Nikolic et al. [32] propose the implementation of an embedded 3D vision-based (2 cameras) SLAM based on bundle adjustment on a heterogeneous Zynq FPGA (hardware + software); the camera's resolution is $752 \times 480$ pixels; the system proposed a hardware implementation of the extraction of FAST and Harris interest points (see $[21,36]$ ), while the rest of the SLAM processing chain is not implemented on the FPGA. The system runs at $20 \mathrm{~Hz}$. Faessler et al. [15] propose the implementation of a 3D optimization-based monocular-dense SLAM on-board a quadrocopter. The camera resolution is $752 \times 480$ pixels, interest points used are FAST corners; the visual odometry chain is implemented on a CPU ARM ${ }^{\circledR}$ (four cores, $1.9 \mathrm{GHz}$ ); dense map reconstruction is implemented on a ground segment (on a CPU i7 $2.8 \mathrm{GHz}$ ). The Wi-Fi communication between the on-board payload and the ground segment limits performances to between 5 and 50 fps (frames per second). In Vincke et al. [46], the authors propose a pure software implementation of a monocular 3D EKF-SLAM processing chain on a multiprocessor heterogeneous architecture - OMAP3530 single chip integrating a RISC processor (ARM ${ }^{\circledR}$ Cortex A8 $500 \mathrm{MHz}$ ) with a SIMD Neon coprocessor, and a DSP (TMS320C64x+, $430 \mathrm{MHz}$ ) Again, the resolution of the cameras is limited to QVGA $(320 \times 240$ pixels). The mean processing times performances announced are around $60 \mathrm{fps}$. 
Considering our scientific and applicative context, these implementations all exhibit part or all of the following drawbacks: limited camera resolution, purely software implementation, 2D (not 3D) SLAM, performances not compatible with ADAS, or involvement of optimization-based SLAM, while our objective is the implementation of a 3D EKFbased SLAM on a heterogeneous architecture, with performances compatible with ADAS constraints. To the best of our knowledge, there exists no implementation of a complete monocular 3D EKF-SLAM processing chain coping with the constraints of an ADAS.

\subsection{Operational vision-based EKF-SLAM implementations at LAAS}

Over the last few years, two operational vision-based EKFbased SLAM processing chains have been developed at the LAAS-CNRS laboratory.

Davison et al. [12] and Roussillon et al. [37] implement a 3D EKF-SLAM processing chain called RT-SLAM (standing for real-time SLAM). Sensors used are an IMU and a single camera (VGA resolution, $640 \times 480$ ). It is a pure software implementation (in $\mathrm{C}++$ language) running on an Intel Core $i 7 \times 86$ desktop processor. The number of landmarks in the environment map and the number of landmarks updated at each image acquisition are configurable. An active search strategy associated with a Harris features detector [21] allows observing, tracking and matching corners. Newly observed landmarks are initialized in the environment map using a classical one-point RANSAC (random sample consensus) algorithm [11]. The global processing time performances reach $60 \mathrm{~Hz}$, but consumption is too high for the ADAS context ( $140 \mathrm{~W})$.

More recently, C-SLAM, based on RT-SLAM and presented in Gonzalez et al. [20] was developed in C language; this software implementation did not use any external software library anymore (as opposed to RT-SLAM). In Botero et al. [6], the authors implement C-SLAM on a dual FPGA platform including a Virtex 5 and a Virtex6 FPGAs. Performances reach $24 \mathrm{~Hz}$ (with $640 \times 480$ image resolution) while observing up to 20 landmarks in each acquired image (up to $20 \times 24$ landmarks updates/s). The C-SLAM implementation allowed to validate the software architecture presented in Sect. 3. The first three layers are in charge of the scheduling of the vision-based EKF-SLAM steps, while the low-level layer involves the computationally intensive operations (the latter layer is where the performance improvement opportunities should be sought for). We have used this functional architecture as a starting point for the works presented here; the initial behavioral model used in our co-design approach (see Sect. 4.2) has been directly derived from this architecture (Fig. 3).

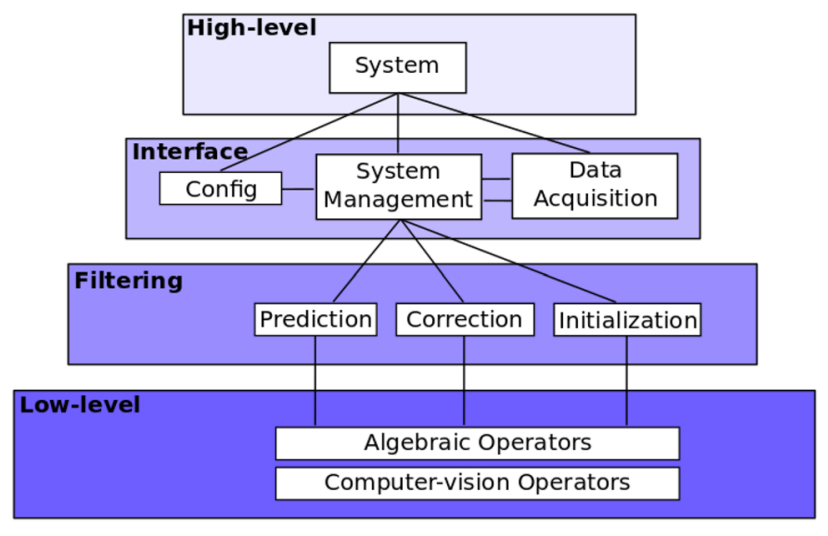

Fig. 3 Architecture of the C-SLAM software [6]

\subsection{HW/SW co-design methodologies}

In the literature, many works related to the development of suitable methodologies for the prototyping of an embedded system can be found. A subset of these refers to heterogeneous architectures and they can be classified [38] as: (A) top-down approaches, where the target architecture is gradually generated from the behavioral system specification by adding implementation details into the design; (B) platformbased approaches, where a predefined system architecture is used to map the system behavior; and (C) bottom-up approaches, where a designer assembles the final hardware architecture by inserting wrappers between operational heterogeneous components.

These methodologies can be referred to as co-design methodologies [mainly for approaches (A) and (B)] or design space exploration (DSE) [for approaches (A), (B) and (C)]. In the following, we explore existing methodologies for co-design; most methodologies in the literature are topdown approaches, that use an application model to specify the application behavior and structure (and thus constitute model-driven methodologies).

The model-driven design flow for the design space exploration (DSE) is a long-studied problem and has proved worthy in a number of applicative domains where the complexity of the targeted application and of the computing architecture may lead to a difficult design process.

In Bezati et al. [3] and Pelcat et al. [34], top-down approaches use the data-flow model of the application to ease the parallelism exploration to target heterogeneous multi-core architectures. Other authors use a high-level programming language that implements a data-flow model of computation that is to be automatically transformed into a valid hardware implementation or multi-core software, see $[41,49]$. Some works rely on the UML model with its architecture specific extensions (MARTE, UML 2.0 see [25]...) 


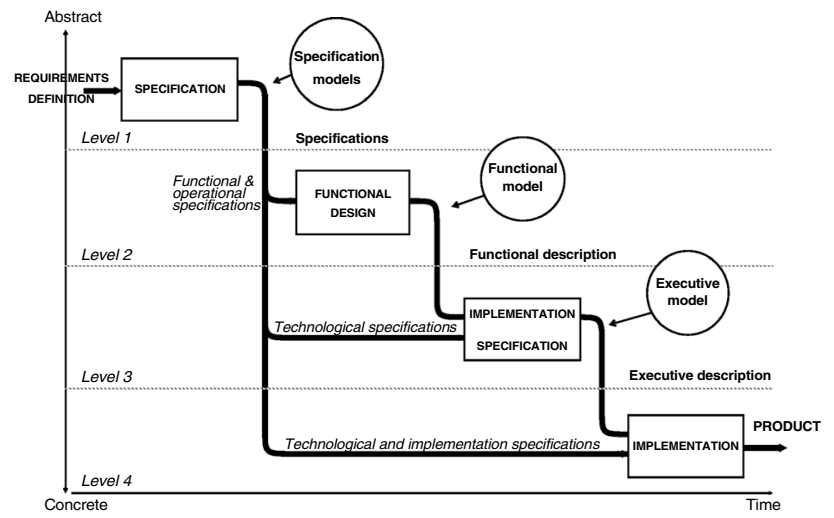

Fig. 4 MCSE prototyping workflow

to study an application and its supporting architecture in the same framework.

Other top-down approaches such as [23] or [9] only define the high-level methodology to be applied for DSE but leave the choice of the languages and the tools to the designer. Methodologies such as the one proposed in Calvez et al. [9] (see Sect. 4) guide the user from the input highlevel specification to a valid system on a chip by imposing a number of intermediate steps (each step being associated with its local model and tests) that follow a V-chart flow. Methodologies such as the one proposed in Kienhuis et al. [23] only define a very coarse design flow from the application and architecture models to a mapping that meets the application constraints. An iteration of the flow maps the application model onto the architecture model and evaluates the performance of the mapping in a simulated environment. The architecture model is then refined until the application constraints are met.

All these methods and tools target the same goal: defining an optimal architecture (with respect to input constraints and input models) for a specific application through the design space exploration. Every method has domain-specific attributes and constraints that may not fit our needs. In Sect. 3, we will propose a generic design flow, refined from Shaout et al. [38], and how we used it to guide our application development.

\subsection{Synthesis: working hypothesis, scientific approach and contributions}

In this paper, we aim at defining and validating a heterogeneous hardware/software architecture for the integration of a complete monocular vision-based 3D EKF-SLAM processing chain meeting the constraints of an ADAS. In Strasdat et al. [42], the authors show that filtering-based approaches appear beneficial for small processing process budgets, as is the case in our study (integration of a monocular sparse vision-based SLAM chain, where a limited number of interest points will be tracked). Furthermore, with optimisation-based approaches, latency cannot be guaranteed, while the number of operations involved in one EKF state update step is deterministic for a given state vector dimension.

Our SLAM-related working hypothesis (derived from the RT-SLAM, see [37], and C-SLAM, see [20]) is as follows:

- we use an IMU system for vehicle motion estimation in the SLAM prediction step;

- we use a single camera (resolution: $640 \times 480$ pixels) for exteroceptive perception;

- we initialize up to five new landmarks in the map for each acquired image;

- we integrate up to 20 landmarks observation in the SLAM correction step;

- we use a corner detector to extract sparse interest points; the process involves a tessellation of the acquired images to provide a better distribution of the extracted interest points in each image;

- the tesselation grid uses $40 \times 40$ pixels tiles resulting in a $16 \times 12$ grid (see Sect. 6.1).

Efficient embedded systems must provide high computational performances and low consumption [19]. The applicative ADAS-related context and the cooperative DICTA project (see Sect. 1) have helped to define the performance target constraints on our system. Frequency requirements are set to a minimum of $30 \mathrm{~Hz}$ (or frames per second). The constraints on the latency of the complete processing chain are set to at most 1 image. We also aim at keeping the total system consumption lower than $5 \mathrm{~W}$ and the design footprint as small as possible (within $25 \mathrm{~cm}^{2} \times 2 \mathrm{~cm}$ ) since we should be able to embed the final SLAM implementation on-board an iCam smart camera, see [13]. None of the operational vision-based SLAM implementations presented in Sects. 2.4 and 2.5 meets these constraints.

The choice of the target platform is linked to the choice of the associated processors. CPUs and GPUs (and to a lesser extent DSPs) provide a much easier implementation of complex algorithms than FPGAs (based on reprogrammable logics and leading to much higher implementation complexity and time). But FPGAs generally lead to far better real-time processing (namely latency and throughput) and power consumption performances, see [17, 35]. When tackling the implementation of embedded complex algorithms (here, dealing with advanced image processing), heterogeneous hardware/ software architectures combining a CPU and a FPGA may allow efficient trade-offs; the algorithms of higher complexity, processing low volumes of data or called at low frequencies may be implemented on the CPU; 
algorithms which are more systematic and/or process high data volumes (typically here, low-level image processing algorithms applied to large number of pixels) can be efficiently and massively parallelized on a FPGA. We chose to implement our embedded SLAM chain on a Zynq-7020 FPGA, only Xilinx ${ }^{\circledR}$ heterogeneous platform providing a heterogeneous architecture combining a CPU (ARM ${ }^{\circledR}$ dual-core Cortex A9 processor) and a FPGA, at the start of the works described here. We chose Avnet's ZedBoard environment for the prototyping of our 3D EKF visual SLAM SoC; this low-cost platform provides all interfaces functionalities for a $\mathrm{PC}$, a monitor, and a USB camera, and relevant processing and memory resources. In particular, it provides an energy-efficient multi-core ARMv7 processor (667 MHz), a Zynq-7020 FPGA device, 512MB DDR3 RAM and a Xillinux [47] distribution which comes quite handy for our needs as it allows memory-mapped and streaming interfaces between the logic fabric and the host processor(s) at no additional engineering effort.

In the following, we describe our implementation of a complete monocular 3D EKF-SLAM on a heterogeneous architecture. Our contribution is threefold:

- we have selected in the literature a (standard and generic) co-design approach which we have (1) refined to allow the integration of a complex processing chain such as a vision-based monocular SLAM and (2) adapted to encompass a hardware-in-the-loop approach allowing to integrate hardware accelerators of advanced image processing or algebraic data processing functions involved in the filtering process. In our approach, the hardware accelerators are integrated one by one. The HIL progressive integration of hardware accelerators is made by using a systematic rule on the results of the cost (here, latency and throughput) profiling of the hardware and software functional components of the heterogeneous SLAM chain under construction.

- we have used this refined co-design method to integrate the first complete monocular extended Kalman filter (EKF) SLAM chain on a heterogeneous (hardware/software) architecture on a single SoC. The obtained processing chain performances meet the constraints of the processing times, power consumption and design footprint of the ADAS.

- we have designed several original hardware accelerators reusable for any embedded SLAM application or vision application involving the same functions. Indeed, we have proposed and validated original hardware accelerators of all the image processing functionalities involved (extraction of interest points corners and computation of their descriptors, tessellation of the images for a better distribution of the interest points all over the images, matching and tracking of the interest points over the sequence of images). We also have developed and validated an original hardware accelerator for the multiplication of matrices involved in the EKF filtering steps.

\section{Proposed co-design methodology}

Co-design is a well-covered scientific topic with solutions ranging from pure design methods to semi-automated design flows. We have identified the following flaws in the existing aforementioned methods (see Sect. 2.6):

- ASIC/SoC oriented design flows do not specify optimizations at the application model level but rather tend to optimize at the implementation level.

- co-design workflows such as in Calvez et al. [9] tend to prefer local iterations at each step (e.g. specification, design, implementation) rather than global iterations (see Fig. 4).

- in design flows such as the one proposed in Pelcat et al. [34], the scheduling partitioning can completely be redefined between two iterations of the same flow with different constraints/scenario input

- design flows such as [34] tend to consider that the designer has an extensive knowledge of the application constraints/properties before prototyping. The flow does not specify how the designer improves the knowledge along the design iterations.

- fully automated design flows (see for example [41, 49]) somehow fail to capture the full extent of the designer's knowledge.

In an effort to get a design flow that simultaneously:

- captures the designer knowledge,

- ensures limited partitioning modifications between two consecutive iterations of the design flow,

- allows for the integration of model-level optimizations,

- ensures IP/software capitalization,

- and allows for HIL performance evaluation,

we came up with the generic workflow depicted in Fig. 5.

This design flow aims at guiding the designer through the DSE but does not include any automatic steps nor is supported by any software tool.

The following subsections describe the workflow steps and then introduce its application to the implementation of our vision-based EKF-SLAM chain. 


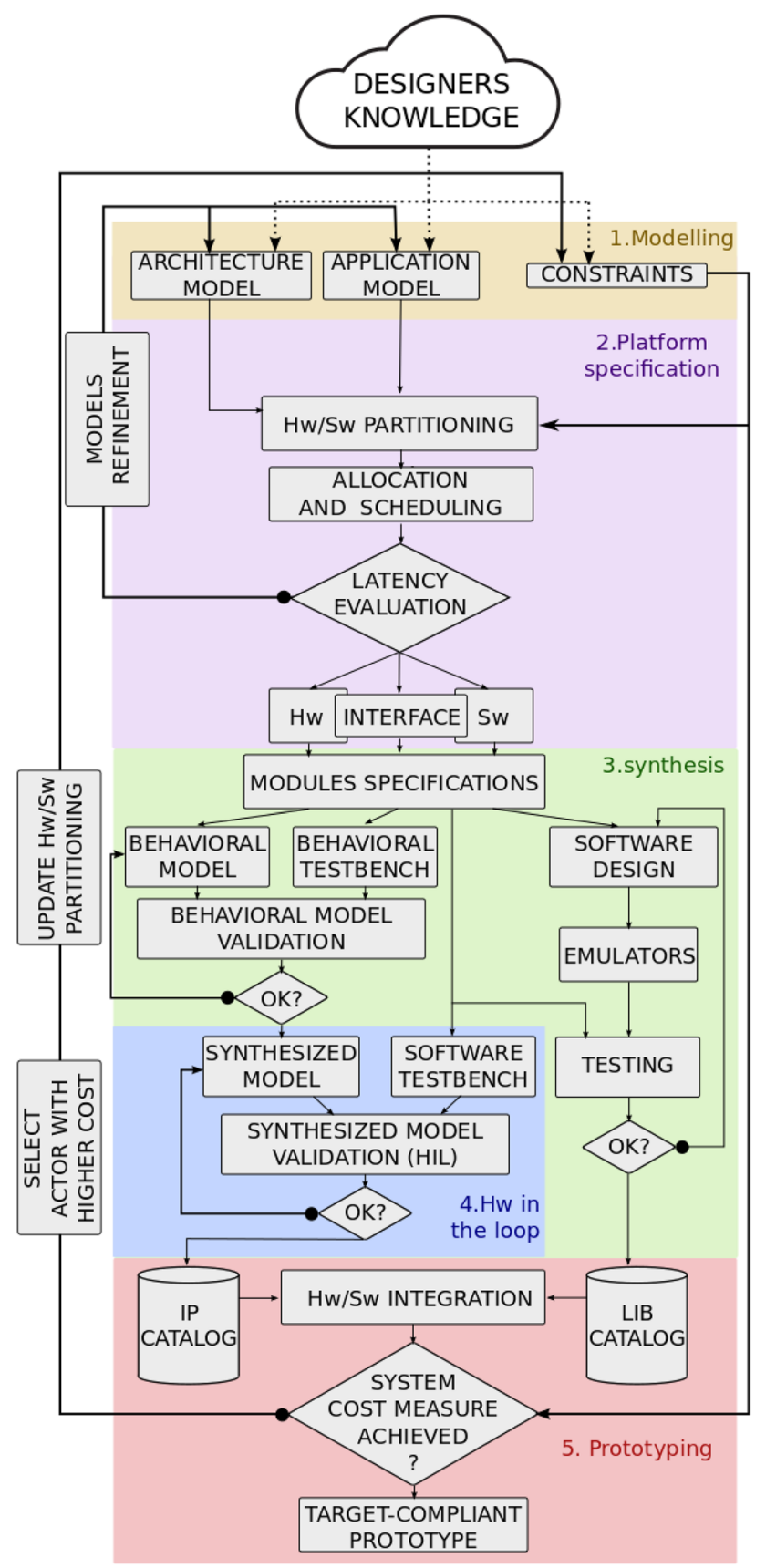

Fig. 5 Proposed co-design methodology for iterative prototyping

\subsection{Design flow description}

The design flow starts with an initial model of the application and either a single-core initial architecture or an architecture imposed by the application. The initial constraints capture the affinity for tasks to be executed on a given processing element and the costs associated with each task (evaluation of task processing time, power consumption, memory footprint ...). In a first iteration, the model is scheduled over the given architecture to evaluate the initial latency (only performance cost that can be evaluated at this step of the co-design method) of the application. This result allows the user to refine the architecture model (i.e., add parallelism degrees) and the application model (i.e., expose more potential parallelism) iteratively, over steps 1 and 2. Once the architecture and application model is considered valid regarding the target latency, the designer can move forward through the prototyping process.

The next step (synthesis) aims at building a softwareonly functional prototype of the application. The designer validates each of the software components using unit-testing techniques. Each of the validated blocks then contributes to a software libraries catalog, to be used for the initial prototype. This initial prototype implements the schedule defined earlier together with the communication primitives for interprocess communication.

This initial software prototype is evaluated against the actual performance defined in the constraints: it is profiled to evaluate the component costs. If the target performance is not met, the actor with the highest cost is selected for optimization. The design flow thus loops back to the Hw/ Sw partitioning with the affinity of the selected actor set to hardware in the constraints. Once an actor is implemented in hardware, the current stage prototype performance is evaluated using an HIL approach. The hardware accelerators are integrated one at a time: a single accelerator is integrated at each global iteration of the HIL process. This design loop keeps going until the prototype meets the target cost.

Similarly to state-of-the-art co-design methods, the proposed design workflow guides the platform designer from model-based specification to a prototype that meets the application constraints. It goes through a number of prototyping steps with local iterations to achieve locally required performance.

The main contributions of this workflow are that:

1. optimizations are performed at the model level and at the implementation level, whereas some methods only consider the model level in the design flow;

2. a systematic decision rule is used to improve the application's partitioning;

3. knowledge is improved at each iteration through profiling;

4. partitioning is modified one task at a time;

5. implementation costs can relate to different attributes (throughput, latency, power consumption) to cover different application domains.

\subsection{Design flow applied to our vision-based EKF-SLAM application}

In the following, we describe the design iterations we followed to conduct the development or our embedded 


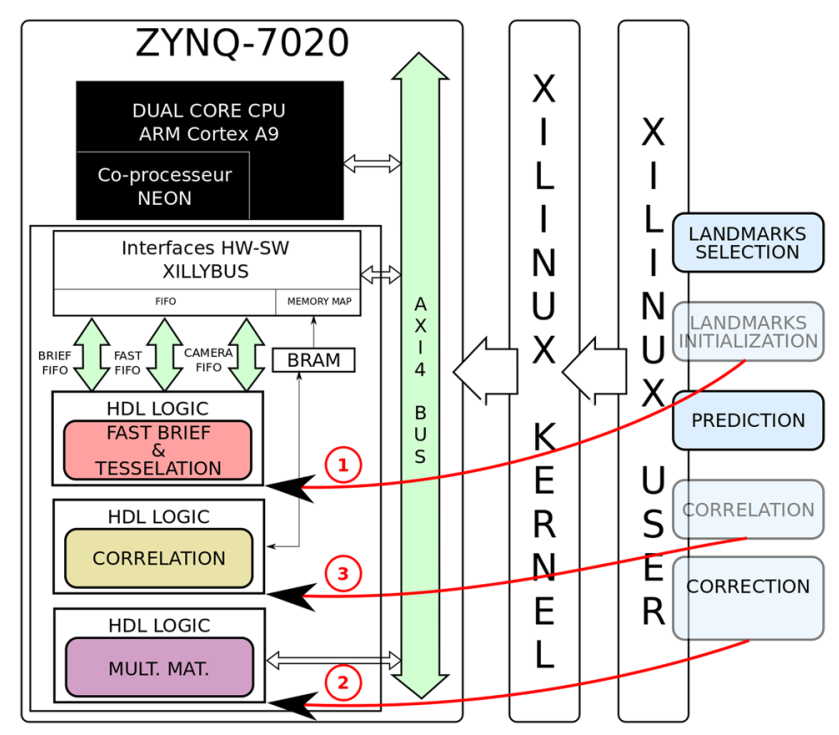

Fig. 6 Iterations for our co-design flow applied to our hardware prototype; each red arrow represents the accelerators successively integrated in our HIL approach

real-time vision-based EKF-SLAM implementation. Section 4 describes the modelling of the design flow inputs. In Sect. 5.1, we detail the optimizations at the application model level for the platform specification step. Then we will successively integrate hardware accelerators (vision-related processes and algebraic operator) to meet the targetted costs (see Sect. 6) as depicted in Fig. 6. The evaluated costs in our application will be the throughput to meet our target frame rate, the design footprint and the power consumption of the system (see Sect. 4.3).

\section{Architecture, application and constraints modelling}

This section deals with the application of step 1 of our codesign methodology, named "Modelling". This stage establishes the architecture model (see Sect 4.1), the application model (see Sect. 4.2), and the constraints on the system (see Sect. 4.3).

\subsection{Architecture model}

As explained in Sect. 2.7, we target an architecture with general purpose processing capabilities and room to integrate hardware accelerators. We chose the Zedboard ${ }^{\circledR}$ development platform based on the ZynQ ${ }^{\circledR}$ Soc that features the following attributes:

- an energy-efficient dual-core $\mathrm{ARM}^{\circledR} \mathrm{v} 7$ processor $(667 \mathrm{MHz})$;
- 512 MB DDR3 RAM;

- 80k logic cells, with DSP and memory blocks;

- a Xillinux [47] Linux distribution which comes quite handy for our needs as it allows memory-mapped and streaming interfaces between the FPGA and the host processor(s) at no additional engineering effort.

This development platform does not meet our physical footprint constraint of $25 \mathrm{~cm}^{2} \times 2 \mathrm{~cm}$, but our design has the potential to be shrinked to this size. While the onboard SoC can have a larger power consumption than the target $(5 \mathrm{~W})$, we will optimize the logic and software to meet that limit.

Our architecture model is composed of two CPU with available logic to host our custom hardware accelerators.

\subsection{EKF-SLAM application model}

The visual EKF-SLAM chain can be described by the following pseudo-code, that we formerly used for our C-SLAM implementation, see [20]:

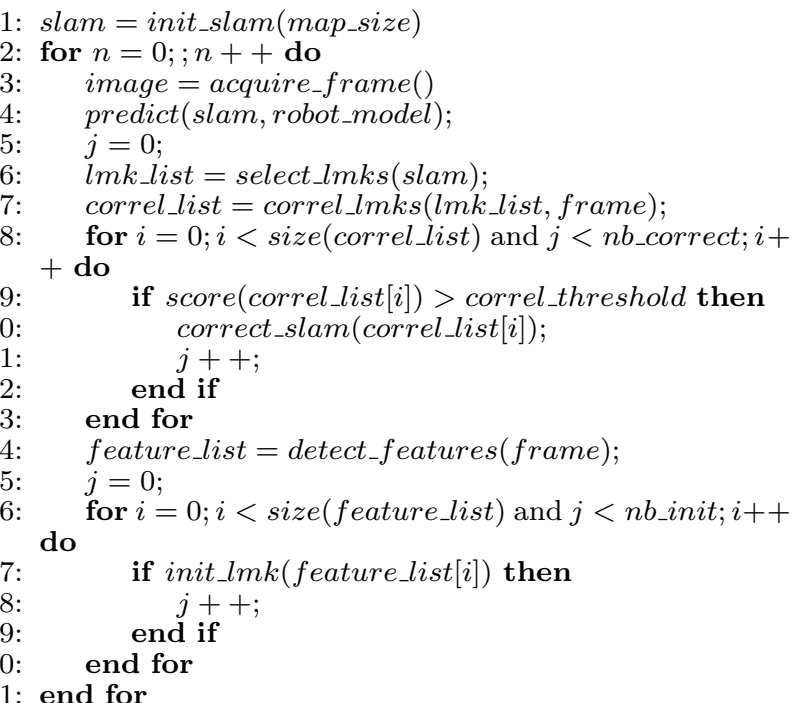

where:

- slam is the global structure that holds the SLAM state and additional information;

- lmk_list is a list of landmarks selected in the environment map for tracking;

- correl_list is the list of observations of these selected landmarks in the current image;

- feature_list is the list of newly detected interest points in the current image; 
and:

- slam_init() is a function that initializes the SLAM state given a map size;

- $\operatorname{predict}()$ is a function that performs the prediction step of the EKF filter;

- select_lmks() is a function that returns a list of landmarks to be observed in the current image;

- correl_lmks() is a function that observes (matches) the selected landmarks with interest points in the current image;

- correct_slam() is a function that performs the correction step of the EKF filter;

- detect_features() is a function that detects interest points in the image;

- init_lmk () is a function that initializes landmarks corresponding to newly detected interest points (landmark initialization). The function returns 0 if the environment landmark map is full.

This pseudo-code can be configured with the following parameters:

- correl_threshold, the minimum correlation score for a landmark to be integrated to the correction step;

- map_size, that defines the total number of landmarks to be managed in the environment map;

- $n b \_c o r r e c t$, that defines the number of EKF correction to be executed per frame;

- $n b \_i n i t$, that defines the maximum of new landmarks to be integrated into the environment landmarks map per frame.

In our C-SLAM implementation, those parameters take the following values:

- $n b \_c o r r e c t=6$,

- $n b \_i n i t=5$.

This C-SLAM algorithm is then adapted to run in an SDF (deterministic) manner. We obtain the SDF model presented in Fig. 7.

\subsection{Constraints}

We remind here briefly the targeted performance contraints expressed in Sect. 2.7:

- SLAM throughput: $30 \mathrm{~Hz}$ (30 fps);

- SLAM latency: 1 image;

- power consumption: $5 \mathrm{~W}$;

- small physical footprint (within $25 \mathrm{~cm}^{2} \times 2 \mathrm{~cm}$ ).

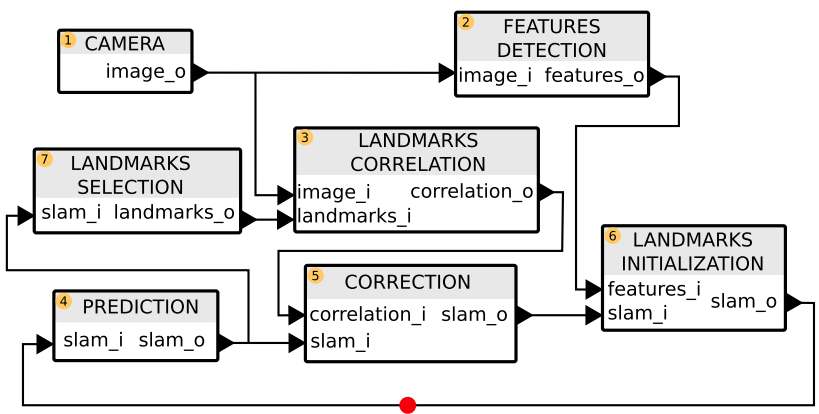

Fig. 7 The vision-based EKF-SLAM SDF model

Now that all the required design flow inputs are defined, we can start iterating the design flow, starting with the model level optimization.

\section{Iteration 1: initial platform specification and model refinement}

As we explain in this section, the initial model does not expose a lot of parallelism and we are not satisfied with the initial latency evaluation; thus we decide to optimize the schedule latency at the model level (see Sect. 5.1). Then, we present the software-only obtained prototype assessment (see Sect. 5.2).

\subsection{Application model refinement}

In SDF applications models, more parallelism can be exposed at the structural level by applying re-timing techniques. Re-timing consists of adding delay tokens on the graph edges to modify the application pipeline.

Such modifications do not affect the structural aspect of the model (the way that nodes are connected) and the schedule length, since the repetition vector of the graph is preserved, but the latency of schedule can be positively affected.

In our 3D EKF-SLAM application, re-timing was achieved by delaying the prediction, correction and landmarks initialization tasks (starting from the SDF model presented Fig. 7, these tasks are delayed in Fig. 8).

Before this re-timing, the directed acyclic graph (DAG) of the application is defined as shown in Fig. 9 (on the left of the picture), with only Feature_detection (2) and Landmarks_correlation (3) to be executed in parallel and a parallelism level of 1.16 (parallelism $=T_{1} / T_{\infty}$ with $T_{1}=7$ and $T_{\infty}=6$ ).

After re-timing, the DAG of the application is defined as shown in Fig. 9 (on the right of the picture), where the filtering [Correction (5)] and [Landmark_initialization (6)] tasks are allowed to be executed in parallel of the vision 


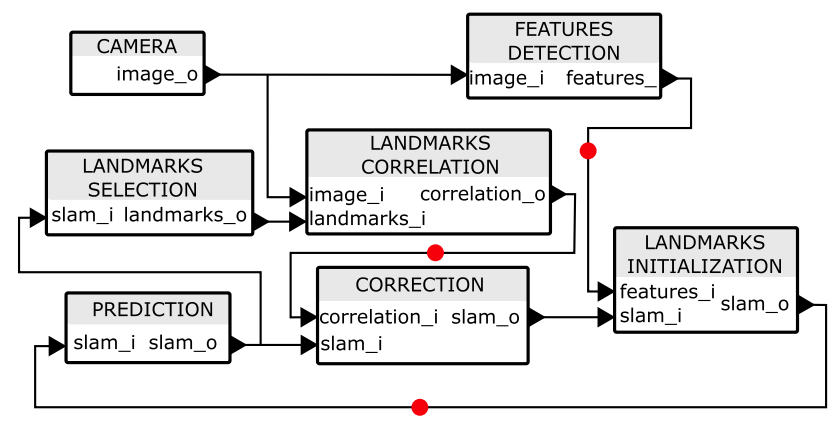

Fig. 8 Re-timed application SDF model

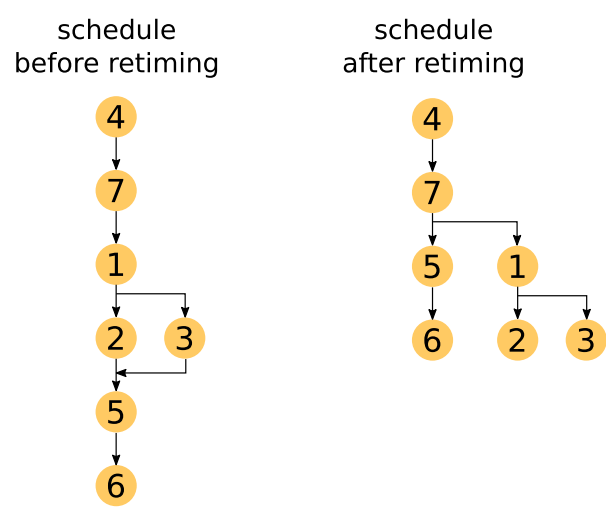

Fig. 9 Application DAG before and after re-timing

tasks. Parallelism level is now 1.75 (Parallelism $=T_{1} / T_{\infty}$ with $T_{1}=7$ and $T_{\infty}=4$ ).

The application now exposes three-degree parallelism. This allows creating a clear partitioning between the filtering tasks (landmarks selection, prediction, correction, landmarks initialization) and the vision tasks (feature detection, landmarks correlation). Re-timing may affect the SLAM's performance since the behavior of the tasks must take the re-timing into account, but is necessary to meet the execution time constraints on a heterogeneous architecture. In this case, the designer should take into account the behavior of the landmarks correlation task as its function is to find landmark matches between the past and the current frame. In cases of, e.g., sudden changes in the orientation, it is highly likely that we "lose" a large number of landmarks. Given the fact that the correction task operates on landmarks from the precedent frame, the quality of the prediction may be corrupted in case of a low number of (or no) landmark matches. Thus, a larger number of landmarks needs to be correlated.

In conclusion to this subsection, we generalize our optimized model as a filtering-based visual SLAM application that stands:

- whatever the filtering technique involved in the correction task is

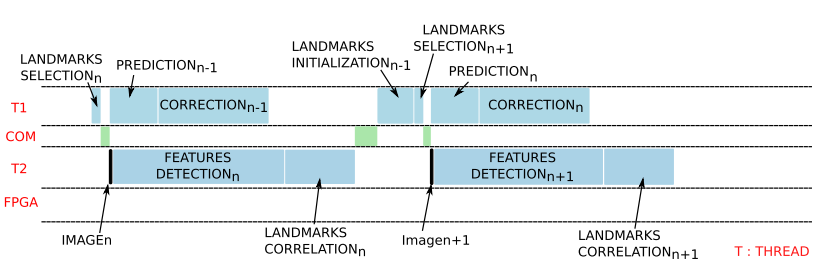

Fig. 10 Schedule of the SLAM application after first iteration

- whatever the feature detection and correlation techniques involved in the features detection and landmarks correlation tasks are

in a filtering-based visual SLAM application.

\subsection{SW-only prototype}

After the model-level optimization, the model is mapped onto the ZynQ ${ }^{\circledR}$ dual-core architecture with all actors affinities set to software. The prototype consists of two threads $T_{1}$ and $T_{2}$ with appropriate synchronization and communication happening through software FIFOs (see Fig. 10).

Vision and filtering tasks are being allocated (mapped) onto two different threads. At this stage, there is no task mapped on a reconfigurable hardware device (FPGA); the entire application is running on the host processor.

This software-only prototype does not match our target throughput (see Table 5). The actor with highest computing cost is identified as the features detection and its affinity is set to hardware in the application constraints.

\section{Iterations 2-4: hardware accelerators}

This section presents the hardware accelerators developed along the iterations of our HIL-oriented co-design methodology (see Fig. 24). We remind that in our co-design methodology, hardware accelerators are integrated one at a time (a single accelerator is integrated at each global iteration of the HIL process). The choices of the successive functionalities to be accelerated are made using the following systematic rule: the costliest function (as far as computing cost is concerned) is the next to be implemented on hardware. The main iterations will be summarized at the end of the codesign process (see Fig. 24).

Our main contributions here are the following FPGAbased original hardware accelerators:

- an accelerator for the features detection task (front endsee Sect. 2.1-vision task accelerator including the hardware implementation of the FAST interest points associated with an image tesselation process), implemented in iteration 2 and presented in Sect. 6.1; 
- an accelerator for the correction step of our EKFSLAM (back end-see Sect. 2.1-filtering task accelerator), developed during iteration 3 and presented in Sect. 6.2;

- an accelerator for the correlation task (front-end vision task accelerator) of our EKF-SLAM, developed during iteration 4 and presented in Sect. 6.3.

\subsection{Iteration 2: feature detection $\mathrm{hw}$ accelerator}

Image features detection is the task of detecting, in images, interesting image structure that could arise from a corresponding interesting scene structure, e.g., a landmark in the scene. Image features can be interest points, curve vertices, edges, lines, curves, surfaces, etc., (see [16]). Our vision-based EKF-SLAM implementation relies on the detection and tracking of corners in an image sequence. The corner detection methods presented in Harris and Stephens [21] and Shi et al. [39] analyze the gradient in the local neighborhood of the potential corner in the processed image through a structure tensor. In Bay et al. [2] and Lowe [28], a difference of Gaussian (DoG) is used to detect a corner. Some of these corner detection methods were successfully implemented on FPGA (see $[4,48]$ ), but the resource count (memory and logic blocks) can be fairly high . FAST (feature for accelerated segment test, see [36]) is a corner extraction method with good corner extraction performances and a very low software complexity. This method is also well suitable for hardware implementation with a very low resource count and no use of DSP blocks, see [24].

There are very few implementations of feature detection modules on a dedicated hardware architecture in the literature. Birem and Berry [4] have implemented a Harris point detector on an Altera ${ }^{\circledR}$ Stratix $I^{\circledR}$ target. But the Harris detector is implemented on (many) DSP blocks of the component. Nikolic et al. [32] have proposed an implementation of Harris and FAST points detectors on a Xilinx ${ }^{\circledR}$ Spartan $6^{\circledR}$. A pixel is processed over four system clock ticks, which limit the input pixel stream frequency. Kraft et al. [24] have implemented a FAST point detector on a Xilinx ${ }^{\circledR}$ Spartan $3^{\circledR}$. The feature detector is executed at 130 Mpixels per second (corresponding to almost 500 frames per second for an image resolution of $512 \times 512$ ), which meets our ADAS-related constraints (as far as throughput is concerned).

In this subsection, we present the design and implementation of our hardware accelerator for the FAST feature extraction, based on the implementation proposed by Kraft et al. [24] and optimized by us (see [7]) to achieve a better maximum frequency (thanks to a pipelined adder tree) and memory consumption (only one 36k BRAM used).

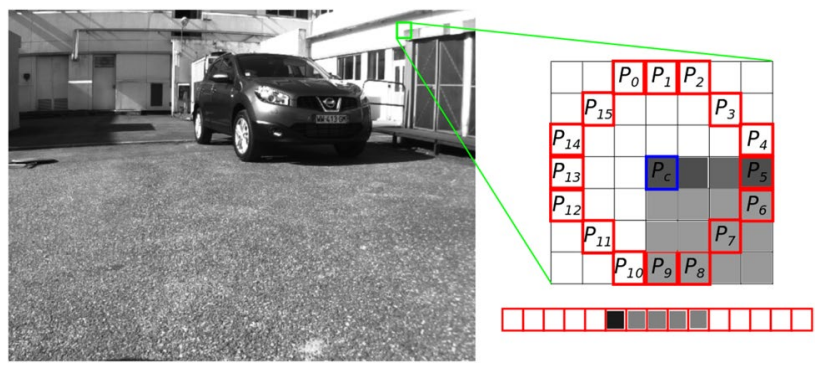

Fig. 11 Fast corner detection principle on sample image used in a SLAM application

\subsubsection{FAST corner detector}

The FAST corner detection at a given candidate pixel of an image (called reference, current or central pixel here) relies on the analysis of the local gradient between this reference pixel and the pixels located on a Bresenham circle centered on the reference pixel (Fig. 11). Let $P_{i}\left(i \in\left[0, \mathrm{BC}_{s}-1\right]\right)$ be the pixels located on this circle, where $\mathrm{BC}_{s}$ is the size of the Bresenham circle (i.e., the number of pixels on the circle). Pixels located on the circle are classified into two classes (darker or brighter) according to the following conditions :

Class $_{P_{i}}= \begin{cases}\text { darker } & I_{P_{i}} \leq\left(I_{p}-t\right) \\ \text { brighter } & \left(I_{p}+t\right) \leq I_{P_{i}},\end{cases}$

where $p$ is the reference pixel, $t$ is a threshold, $I_{p}$ is the intensity of the reference pixel and $I_{P_{i}}$ is the intensity of a given pixel on the Bresenham circle. If a set of minimum $N$ contiguous pixels are classified as brighter or darker, then $p$ is classified as a corner. The detector has got three parameters: the Bresenham circle size $\mathrm{BC}_{s}$, the threshold $t$ and the minimum number of contiguous pixels $N$.

Figure 11 shows an example of a corner with the corresponding Bresenham circle.

Once a corner is detected, its score, given by Eq. 5, is computed.

$V=\max \left(\sum_{P_{i} \in \text { brighter }}\left|I_{P_{i}}-I_{p}\right|-t, \sum_{P_{i} \in \text { darker }}\left|I_{p}-I_{P_{i}}\right|-t\right)$

Figure 12 presents the global architecture of our hardware accelerator for the FAST feature detection. The FAST hardware implementation is composed of four processing blocks: Thresholder, Corner Score, Contiguity and Score Validation.

The Thresholder processing block uses threshold $t$ to compare the central pixel to the pixels on the circle, and provides two vectors of $\mathrm{BC}_{s}$ bits each indicating whether the successive pixels on the circle are, respectively, brighter or darker than the central pixel. These two 


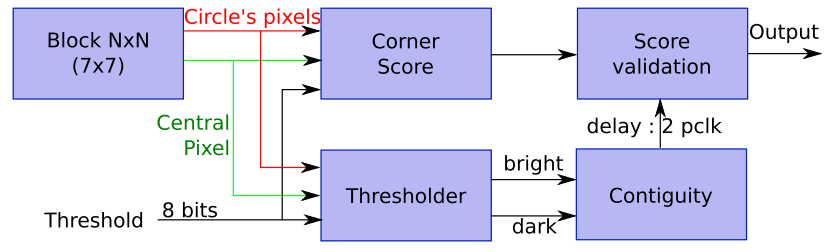

Fig. 12 FAST architecture

vectors are used by the CornerScore block, that computes the current pixel score $V$. The Contiguity block determines whether at least $N$ contiguous pixels are brighter or darker than the central pixel. There are $\mathrm{BC}_{s}-N$ successive arcs of $N$ contiguous pixels on our Bresenham circle; for each of these arcs, $N$ comparisons are needed to check whether all pixels belong to the same (brighter or darker) class. Therefore, our Contiguity block uses $\mathrm{BC}_{s}-N$ comparators, each of them performing $N$ comparisons. All comparisons are performed in parallel. If at least one of the contiguity tests performed by the $\mathrm{BC} s-N$ comparators is successful, the presence of a corner and its score are validated by the Score validation block.

The implementation of the four material processing blocks is composed of logic elements performing sums, differences, absolute values and comparisons. The implementation of most of these is quite straightforward. The inputs of the CornerScore and Thresholder processing blocks are the central (current) pixel, the pixels of the Bresenham circle, and threshold $t$. Outputs of the Thresholder processing block (and inputs of the Contiguity block) are the elements of the $\mathrm{BC}_{s}$ bits vectors indicating whether the successive pixels on the circle are, respectively, brighter or darker than the central pixel. The main hardware acceleration is provided by the adder tree instantiation which computes pipelined additions. It allows to perform, in our case, additions of eight inputs per clock cycle-with a latency two clock cycles instead of eight clock cycles using a basic processor, see [7].

As far as the pixel pipeline flow is concerned, pixels are stored in a cache (BRAM) allowing to feed the FAST corner detection hardware blocks with the pixels on the Bresenham circle. If $\mathrm{BC}_{d}$ is the diameter of the Bresenham circle, we need a sliding processing region of $\mathrm{BC}_{d} \times \mathrm{BC}_{d}$ pixels in the image; thus, we need to buffer $\mathrm{BC}_{d}$ image lines.

Our hardware architecture is generic. The size of the Bresenham circle $\mathrm{BC}_{s}$, the threshold $t$ and the number $N$ of contiguous pixels needed to detect a corner can be parameterized. The typical values we have chosen are $\mathrm{BC}_{s}=16$, $N=9$ and $t=20$, recommended by Rosten and Drummond [36].

The results of our material architecture implemented for the detection of FAST features are consistent with the state-of-the-art functional implementations (we assessed this
Table 1 Use of the logic resources of the Zynq xc7z20-1clg484 for VGA $(640 \times 480)$ image resolution

\begin{tabular}{llll}
\hline Logic utilization & Used & Available & Utilization $(\%)$ \\
\hline Slice LUTs & 5963 & 53200 & 11 \\
BRAM $(18 \mathrm{k} / 36 \mathrm{~K})$ & $0 / 1$ & $140(38 \mathrm{~K})$ & 0.5 \\
Slice registers & 8281 & 106400 & 7 \\
\hline
\end{tabular}

through a comparison with a software implementation based on the use of the OpenCV library).

On our FPGA target (Xilinx ${ }^{\circledR}$ ZynQ $^{\circledR}$ zc7z20), we obtain a maximum frequency of $160 \mathrm{MHz}$, i.e., our feature detection hardware accelerator can process up to $160 \mathrm{Mpixels} / \mathrm{s}$ (maximum frequency for a VGA image resolution) with a latency of $\mathrm{BC}_{d}$ pixels and $\mathrm{BC}_{d}$ image lines (7 lines for a Bresenham circle of 16 pixels perimeter). This throughput corresponds to the processing of up to 77 frames/second for a full HD image resolution $(1920 \times 1080$ pixels $)$, with a maximum power consumption of $2.5 \mathrm{~W}$ (estimated using the Xilin $x^{\circledR}$ XPower Analyzer ${ }^{\circledR}$ tool). These results bring to light a very good computational power/consumption ratio and validate our FAST interest point extraction hardware accelerator. Table 1 summarizes the use of the resources of the FPGA.

In comparison with the state of the art of FAST feature extraction hardware accelerators, the architecture proposed in [24] uses 12 BRAMs, 2368 LUTs and 1547 registers of a Spartan ${ }^{\circledR} 3$ FPGA, with a maximum processing clock of $130 \mathrm{MHz}$ (for a $512 \times 512$ images). The improvements brought by our architecture are a lower BRAM count and the use of a pipelined adder tree to increase the maximum frequency.

\subsubsection{Tesselation hw accelerator}

The second hardware acceleration of the feature detection front-end vision task is the tesselation of the corner extraction. Tesselation is the process of tiling the image in small blocks using a rectangular grid having Rows lines and Cols columns, to beneficially:

- reduce the image processing complexity by applying image processing techniques only to a subset of image blocks (delimited by the grid cells) instead of processing the entire image;

- avoid the need for the use of a non-maxima suppression technique since we extract a single feature (the one with maximum score) within each tesselation grid cell.

In our original software-only SLAM implementation, this tesselation was used so that at each iteration only five image blocks were selected to compute the corner 
detection (see parameters settings presented in Sect. 4.2) and only one corner per image tile was extracted. This helped reduce the image processing time but also allowed the algorithm to improve the spatial distribution for the corners to be integrated as landmarks into the SLAM map.

Our tesselation module uses a BRAM memory that stores the best score for each tile. Since the image is processed as a stream of pixels, only a single tile is active (i.e., currently being searched) at a time. The score and maximum position for a given block are output when the pixel stream reaches the bottom right corner of the block. The module outputs a stream of scores and corresponding maxima positions (one maximum position and corresponding score per block).

The memory consumption (Mem) of the FAST feature extraction and tesselation hardware modules depends on the number of columns (Cols) of the tesselation grid, the number of bits used to code the $(X, Y)$ position of a pixel with maximum score in a block (nbrbit $X$ and nbrbitY) and the number of bits used to code the score (nbrbitscore). The following Eq. 6 computes the memory consumption:

Mem $=$ Cols $\times($ nbrbit $Y+$ nbrbit $X+$ nbrbitscore $)$.

In our case, Cols $=16$, nbrbit $Y=10$ bit, nbrbit $X=10$ bit and nbrbitscore $=12$ bit . The tesselation hardware module requires only $\mathrm{Mem}=512$ bits.

Our tesselation HW module also implements two counters (a pixel counter and a line counter) which allow defining the current tile (i.e., currently active tesselation grid cell) index and the current pixel position in this tile. A comparator compares the current pixel FAST score to the maximum score in the tile and stores the current score and position in the BRAM only if this score is higher than the current maximum score in the corresponding tesselation grid cell.

Figure 13 shows the tesselation grid, in black, and the highest score in each tile, in color: colors used range from red to blue for an increasingly good feature.

Our tesselation module efficiently helps meeting the processing times constraints for our embedded SLAM chain. Moreover, a feature is detected by the FAST corner detector only one time, during the feature initialization step. Then, by updating the EKF filter, the algorithm predicts the new feature position in the next frame. Thus, the feature is tracked by correlating the descriptor BRIEF in a given and predicted area of research (see Sect. 6.3).

Furthermore, the latency of the FAST feature extraction and tesselation hardware modules is fixed and given by Eq. 7 :

$L=(I M G H / \text { Rows }+(\text { FASTrad }))_{\text {lines }}+1_{\text {pixel }}$,

where $I M G H=480$, Rows $=12$ and FASTrad $=4$ pixels, where $I M G H$ is the number of lines in our images

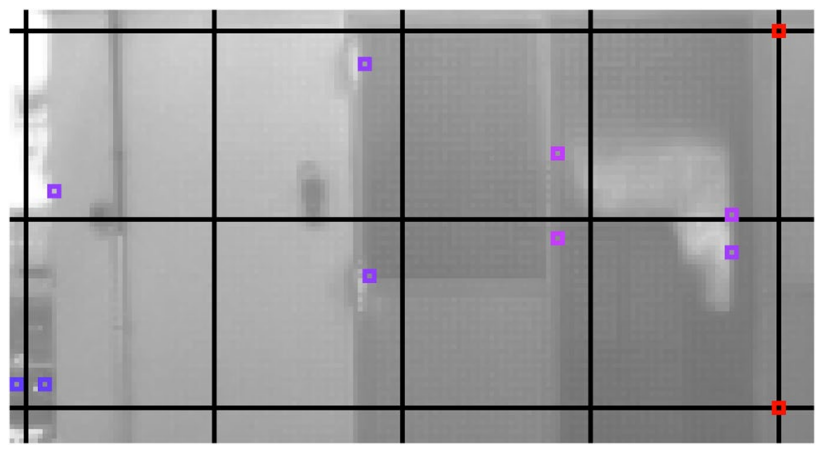

Fig. 13 Tesselation on FAST corner detection result

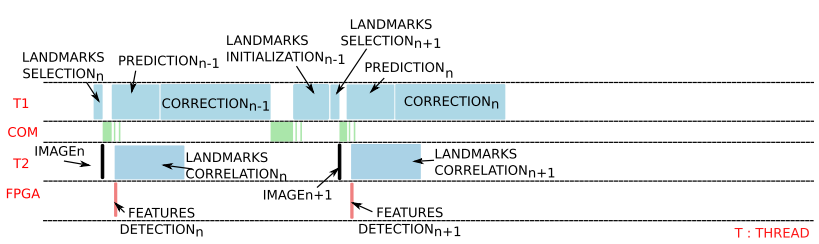

Fig. 14 Schedule of the SLAM application after second iteration

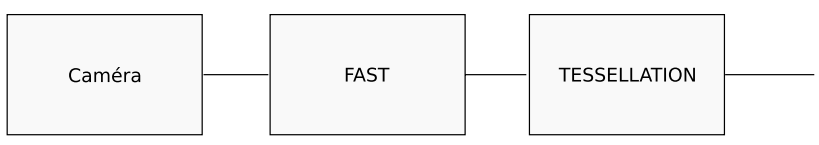

Fig. 15 Image processing pipeline for the corner extraction

and Fastrad is the radius of our Bresenham circle. Latency $=44 \times$ line $_{\text {latency }}+1 \times$ pixel $_{\text {latency }}$.

The processing pipeline of the FAST feature extraction and tesselation hardware modules also transfers back to the software processor a fixed amount $D$ of data which depends on

Rows, Cols, nbrbit X, nbrbitY and nbrbitscore (the number of bits used to code a corner score).

$D=($ Cols $\times$ Rows $) \times($ nbrbitY + nbrbit $X+$ nbrbitscore $)$,

i.e., $D=768$ Bytes in our design.

The new scheduling obtained after the integration of our feature detection and image tesselation HW accelerator is presented in Figs. 14, 15 and Table 5.

\subsection{Iteration 3: algebraic hardware accelerator}

The goal of this section is to give a theoretical background to identify the computational bottleneck of the EKF block used for a visual EKF-SLAM algorithm in general. Afterwards, we will focus on the integration of an adequate EKF accelerator. 
Table 2 EKF matrix description

\begin{tabular}{lll}
\hline Symbol & Dimension & Description \\
\hline$\hat{x}, \hat{x}^{+}$ & $(7 N+r) \times 1$ & Robot and feature positions (actual and predicted) \\
$P, P^{+}$ & $(7 N+r) \times(7 N+r)$ & Cross-covariance matrix (actual and predicted) \\
$P_{1}$ & $r \times r$ & Cross-covariance matrix with respect to robot position \\
$P_{2}$ & $r \times 7 N$ & Cross-covariance matrix with respect to all landmarks \\
$P_{3}$ & $7 \times 7$ & Cross-feature-robot covariance matrix \\
$P_{4}$ & $(7 N+r) \times 7$ & Cross-feature-feature and robot-robot covariance matrix \\
$F_{x}$ & $r \times r$ & Jacobian to system state \\
$F_{\omega}$ & $r \times 6$ & Jacobian to system state perturbation \\
$Z$ & $2 \times 2$ & Covariance innovation \\
$H$ & $2 \times 7$ & Jacobian \\
$R$ & $2 \times 2$ & Measurement noise \\
$K$ & $(7 N+r) \times 2$ & Filter gain \\
$y$ & $2 \times 1$ & Measured output \\
\hline
\end{tabular}

The profiling of our accelerated EKF-SLAM implementation shows that the most time-consuming task is correction_slam. According to our methodology, this task must now be accelerated through the use of hardware IPs.

\subsubsection{Complexity study of extended Kalman filter for the correction step}

The EKF general framework was described in Sect. 2.3; the following Table 2 gives a breakdown of data sizes for the correction step (see Eq. 4), using:

- $N$, the number of landmarks observed in the correction step ( $N=20$ in our implementation);

- $r$ the size of the robot state vector $(r=19$ in our implementation)

\subsubsection{Vision-based EKF accelerator co-design}

In Thrun et al. [44], the authors show that computational requirements for an EKF algorithm depend on the number of features $N$ retained in the map: $L_{\mathrm{EKF}}=O\left(N^{2}\right)$. We can see that the most computationally expensive equations take place in the correction loop while updating the crosscovariance matrix (see Eq. 4) which makes for $85 \%$ of all the floating-point operations (FLOPs) when $N=20$, which matches the profiling of our EKF-SLAM implementation. Thus, a significant speed-up can be obtained by leveraging the floating-point $\mathrm{KZK}^{T}$ tri-matrix multiplication and subtraction operations in hardware.

Another particularity of visual EKF-based SLAM is that the dimension of the covariance innovation matrix $Z$ is always $2 \times 2$. Moreover, in the literature, efficient reconfigurable designs harboring matrix multiplication operations in floating-point precision are processing element
(PE) oriented, see $[22,50]$. Consequently, we can rewrite $P-K Z \times K^{t}$ as a PE-based pseudo-code:

Phase I: $K \times Z$

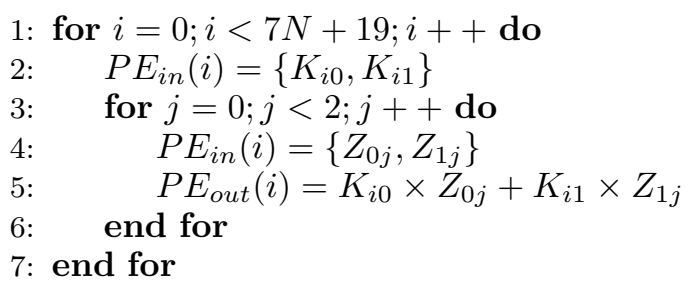

Phase II: $P-K Z \times K^{t}$

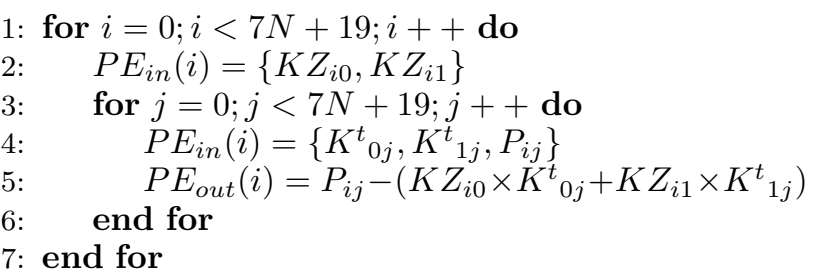

Phase I and Phase II are executed sequentially on the same PEs. Each PE consists of two floating-point multipliers, one floating-point adder (which perform $K Z K^{t}$ ) and one floating-point subtractor (which performs $P-K Z K^{t}$ ). Thanks to the size of the $Z$ matrix, we are able to instantiate multiple PEs that do not have to communicate to each other intermediary multiplication values (on line 5 of both Phase I and Phase II pseudocodes the output function of a $P E$ does not depend on intermediary multiplication values). This design permits subtractions in equation $P-K Z \times K^{t}$ to be executed right after the multiplication under the condition that each corresponding element of the $P$ matrix is already fetched from memory. Thus, not only the tri-matrix 


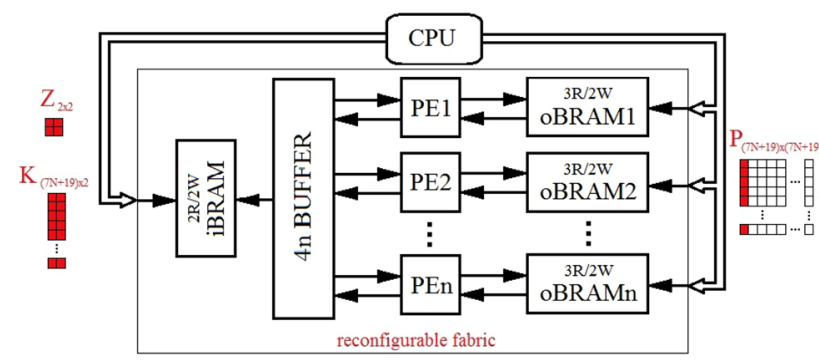

Fig. 16 Generic tiling for $P-K Z \times K^{t}$

Table 3 Transfer analysis regarding $P$ matrix in EKF equations

\begin{tabular}{lccc}
\hline$\% \mathrm{xfers}$ on & $N=6$ & $N=13$ & $N=20$ \\
\hline$F P F_{T}+G Q G^{T}$ & 1.21 & 0.2 & 0.06 \\
$H P H^{T}+R$ & 3.95 & 1.4 & 0.7 \\
$P H^{T} Z^{-1}$ & 17.2 & 11 & 7.96 \\
$P-K Z K^{T}$ & 74.96 & 86.45 & 90.8 \\
\hline
\end{tabular}

multiplication is executed in a pipelined manner, but we also improve the performances of the subtraction as its latency is now the one of a single floating-point subtractor. Results are output column-wise which eases later addressing issues in software, see Fig. 16.

As our entire EKF-SLAM algorithm is designed to run as a SoC on a ZynQ device, we designed and implemented a generic accelerator that takes into account both latency and the aforementioned integration issues. We instantiate four PEs. In terms of latency, performances are close to the theoretical speed-up, see [43]. The reasons for not having instantiated more PEs are tied to resource usage (LUTs for logic and DSP48E embedded multipliers for floating-point calculations), and thus the percentage of the used area on the device which would consequently decrease the maximum operating frequency and/or the power consumption.

Having in mind that square matrix $P$ is the biggest structure in our SLAM algorithm in terms of memory usage, it is imperative to ensure efficient data communication between the accelerator and the rest of the system. In Table 3, we can see the influence of $N^{2}$ in equation $P-K Z \times K^{t}$, as far as the number of element-wise memory accesses is concerned, by changing the value of $N$. An accelerator which would transfer back all the results into external memory (where program data are stored) would suffer from a large memory access penalty with growing $N$. By storing the $P$ matrix in on-chip memory instead of storing it in external memory and by modifying the software to access the contents of $P$ in on-chip memory, we would in addition gradually increase the efficiency of the accelerator.

In each EKF iteration, matrices $P_{1}, P_{2}, P_{3}$ and $P_{4}$ are fetched and updated from on-chip memory via a dedicated

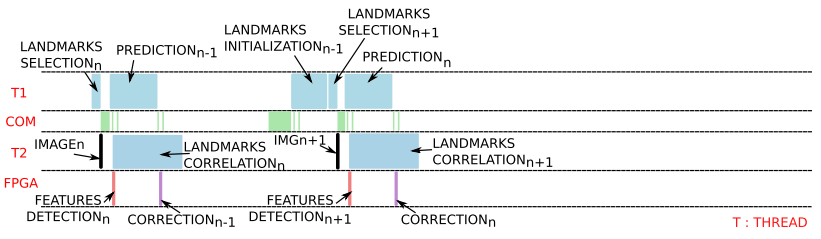

Fig. 17 Schedule of the SLAM application after third partitioning

AXI bus. Then, matrices $Z$ and $K$ are copied from external memory to on-chip memory (iBRAM). Control data are communicated directly from the Xillinux user space to the co-processor. The logic fetches by itself matrices $Z, K$ and $P$, and stores the result back in $P$ (oBRAM) via dedicated XIL_BRAM buses. The processor is notified of the end of execution via an interrupt signal.

After the integration of this back-end accelerator, the 3D EKF SLAM's tasks' timing changes as on Fig. 17.

Since the accelerator IP is generic, our co-design technique may be migrated to bigger reconfigurable devices if there is a demand for more processing power (PEs). Accelerator was also successfully tested as a PLB peripheral for heterogeneous designs on a FPGA with IBM's PowerPC440 embedded processor.

The resulting speed-up when using the algebraic accelerator is listed in iteration 3 of Table 5. This table shows that the next iteration of our design workflow must now target the acceleration of the landmark correlation step.

\subsection{Iteration 4: feature correlation hw accelerator}

In this section, we present an original hardware accelerator for the tracking and matching of features through the input image flow. Our working hypothesis is derived from our initial software C-SLAM implementation, see Sect. 2.7. The landmark map is initialized from the initially detected FAST features. Then, the selected features are tracked in the next images using an active search strategy [20]. The size of the search windows is derived from the innovation covariance of the EKF state. A descriptor-based matching in these searching areas is performed by comparing feature descriptors.

Many approaches to compute feature descriptors can be found in the literature. The Binary Robust Independent Element Feature (BRIEF) descriptor [8] has got many advantages over the other well-known SIFT [28] or SURF [2] descriptors from the point of view of their suitability for hardware implementation. It is indeed a binary descriptor representing the distribution of gradients in the neighborhood of the pixel of interest. First, a $N_{b} \times N_{b}$ patch centered on this pixel is extracted. $M_{b}$ pairs of pixels in the patch are randomly selected (see Fig. 18), and for each pair $p_{0}, p_{1}$, a bit is generated as follows: 

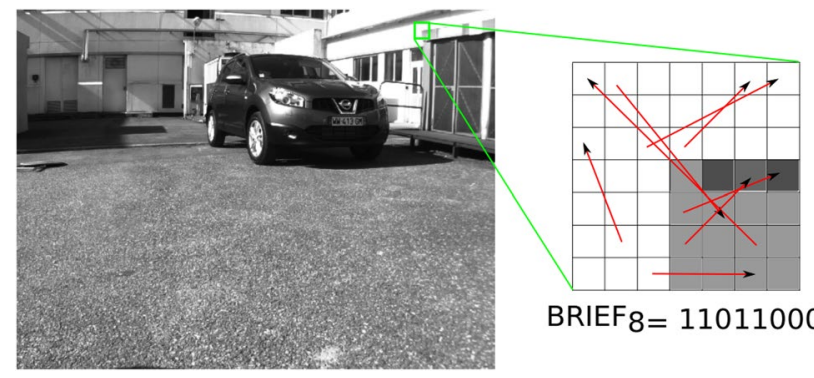

BRIEF $8=11011000$

Fig. 18 Computation of the BRIEF descriptor

$b_{\mathrm{m}}=\left\{\begin{array}{ll}1 & \text { if } p_{0}<p_{1} \\ 0 & \end{array}\right.$.

This results in a $M_{b}$-bit long binary descriptor. The BRIEF descriptor can be computed very fast, is very compact and exhibits good stability for the matching. The descriptor is robust to illumination changes and small rotations (less than $15^{\circ}$ ) [8] which makes it a good choice for SLAM applications. Its simplicity makes it a good candidate for hardware implementation. The comparison of two binary numbers can be done very simply and implemented very efficiently.

For our embedded SLAM implementation, we associate each FAST feature detected in the images to a BRIEF descriptor. We decided to compute 128-bit descriptors in a 9 pixels $\times 9$ pixels neighborhood.

Comparing the BRIEF descriptors of 2 pixels comes down to computing the Hamming distance of two 128-bit vectors. Eqs. 9 and 10 show the two steps of the Hamming distance computation:

tmp $=B R I E F_{\text {ref }}$ XOR BRIEF $F_{\text {current }}$,

HDist $=$ nOnes $(\mathrm{tmp})$,

where $n$ Ones () counts the number of bits equal to ' 1 ' in the tmp vector. Counting the ones in a 128-bit vector proves expensive in term of hardware and latency. To simplify the process, the input 128-bit vector is divided into smaller vectors of size $n$ ( 6 bits in our case) for which the hamming distance can be computed using a $n$-inputs LUT. The result of each sum is then added using a pipelined adder tree. This induces a latency of four clock cycles in our design which in return consumes fewer logic cells than a classical 128-bit Hamming distance computation.

Figure 19 shows a correlator core. Data for correlation $\left(B R I E F_{\text {ref }}\right.$, ROIsize and $\left.X 0 / Y 0\right)$ and the correlation result $(X, Y, B R I E F$ and HammingDistance $)$ are stored in the same BRAM which can be read and written to by both the hardware and the ARM host. A signal result_available is asserted to notify the end of the computation.

In the global image processing pipeline (see Fig. 21), the FAST feature extraction and BRIEF descriptor

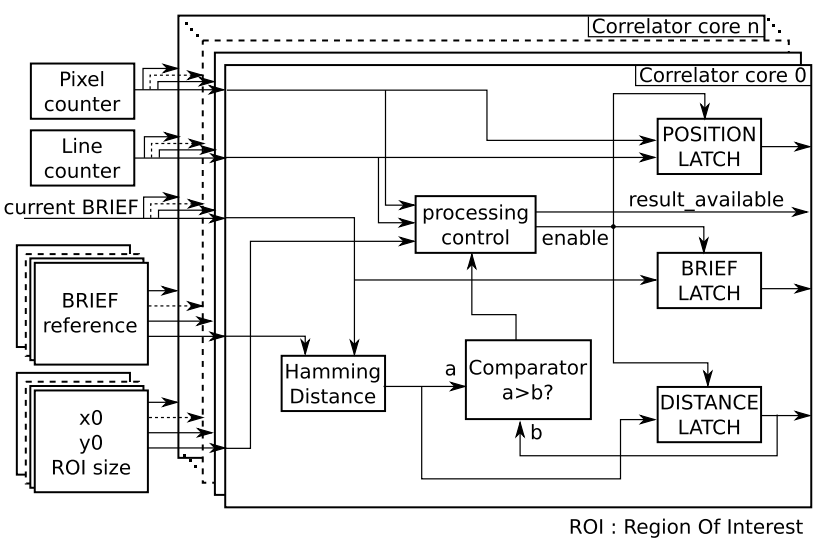

Fig. 19 Instantiation of a multi-core correlator

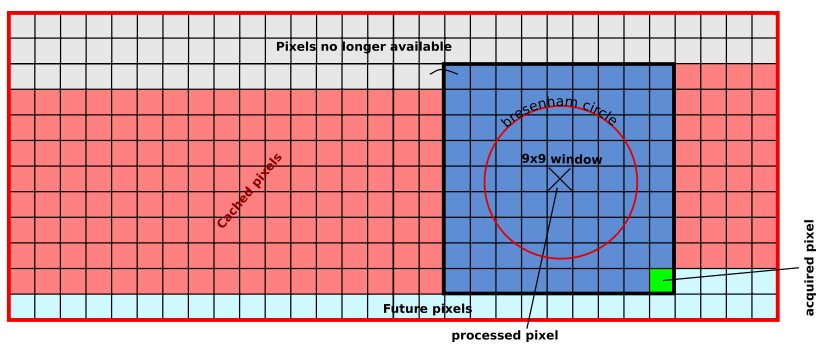

Fig. 20 Cache management for the shared memory between BRIEF descriptor computation and FAST corner extraction. The Blue area depicts the pixel used for the BRIEF descriptor computation. The Bresenham circle for the FAST computation is included in this area

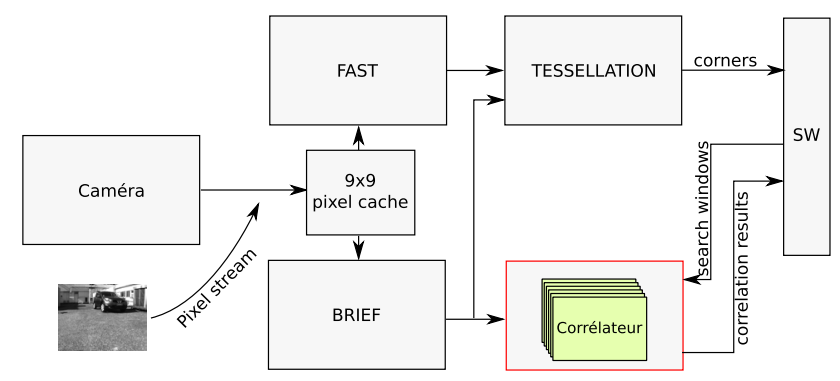

Fig. 21 Image processing pipeline for descriptor extraction and matching

computation are performed in parallel. As several ROIs in which correlation using Hamming Distance is performed may overlap, we decided to instantiate as many correlation cores in parallel as the number of landmarks managed in the environment map (typically 20 in our case as stated in Sect. 2.7). Since the two vision-processing tasks partially depend on the same set of pixels, the cache memory was optimized to store the pixel shared between the two tasks (see Fig. 20). One key advantage of this accelerator is that its throughput is independent from the size of the search 


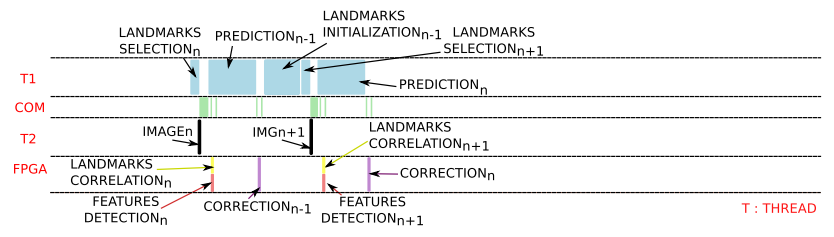

Fig. 22 Schedule of the SLAM application after iteration 4

area since the descriptors are computed and correlated in parallel, on the pixel stream.

In Bonato et al. [5] and Yao et al. [48], respectively, the authors implement the SIFT feature descriptor in hardware. The SIFT feature descriptor is better than BRIEF in terms of stability, but the resource count is much higher from the viewpoint of the use of DSP blocks (respectively, 64 and 97), the use of LUT (respectively, $35 \mathrm{k}$ and $43 \mathrm{k}$ ) and the use of registers (19k for both), and the frame rate is much lower (30 FPS for QVGA images in Bonato et al. [5] and 30 FPS for VGA images in Yao et al. [48]). Moreover, these two hardware designs only compute the descriptor, while the correlation is performed in software. In de Lima et al. [26], the authors use a FPGA to compute a 256-bit BRIEF descriptor. The implementation only considers the descriptor computation and was designed using HLS tools. This implementation is shown to work well for QVGA images stored in memory and processed using a $125 \mathrm{-MHz}$ system clock but no implementation of the correlator is proposed.

Our BRIEF correlator can run at up to a $160.436 \mathrm{MHz}$ pixel clock and uses only $6 \%$ of our ZynQ 7020 registers and $18 \%$ of its LUT. The BRAM consumption depends on the image resolution. For a VGA resolution, only $836 \mathrm{~kb}$ BRAM are used.

After integration of this front-end accelerator, 3D EKFSLAM tasks' timing changes as on Fig. 22.

\section{Final hardware prototype}

This section begins with a description of our visual 3D EKF-SLAM's SoC architecture, at the end of our co-design process (Sect. 7.1). Afterwards, the obtained global experimental results are presented and discussed (Sect. 7.2) and put into perspective with respect to the state of the art (Sect. 7.3).

\subsection{Final hardware architecture}

The developed hardware architecture consists of three IPs instantiated in the reconfigurable fabric of a ZynQ-7020 device: the FAST feature detector and image tesselation module, the EKF module, and the correlator module. All vision tasks and the most time-consuming task in the EKF are executed on FPGA, while the rest is scheduled on ARM under Xillinux (user space). Hardware-accelerated functions are integrated into the embedded system as co-processors. The CPU communicates with our IPs via Xillinux middleware (kernel space) and the Xilly_vga, Xillybus_lite, and Xillybus IPs are also instantiated in the logic, see Fig. 23.

The FAST+Tesselation accelerator is interfaced with Xillinux through Xillybus FIFOs (camera_fifo and FAST_fifos) which are accessed in the user space through files. The first is used for frame pixel reception, which is made from image files in Xillinux. The whole image sequence, and the IMU and GPS data used by our application are pre-registered and stored in external memory. This feature detection logic runs at $89 \mathrm{MHz}$, limited by the maximal throughput supported by the Xillybus IPs. Control signals are communicated from the host through Xillybus_lite, which serves as a memorymapped (host to FPGA BRAM) interface. The correlator module communicates with the middleware as a memorymapped device.

Our modification to this off-the-shelf prototyping architecture was introduced in the third co-sythesis loop by changing the devicetree.dts file so that this architecture could support addressing through an additionally instantiated AXI4 stream bus (namely axi_interconnect_2) for the EKF accelerator $\left(P-K Z K^{T}\right.$ operation). In this way, we were able to set-up a new clock domain $(96 \mathrm{MHz})$ and to facilitate the HIL process. An individual bus is also imperative because of the $P$ matrix-related transfer rates-see Table 3 .

\subsection{Experimental results and discussion}

In Table 4, we present the summary of the Zynq-7020 device's resource usage. The BRAM usage is high because apart from instantiating it for the storage of the $P$ matrix, it is also used for implementing the Xillybus FIFOs (6x BRAM36K). The maximum frequency of individual accelerators is higher than actually set, because of the timing issues of the integrated bare-metal design with the Xillybus IPs. The Xilinx Power Analyzer ${ }^{\circledR}$ is used to measure the power consumption in the reconfigurable logic $(0.671 \mathrm{~W})$-this measure does not include the ARM's power consumption. The power consumption was measured, on the ZedBoard power input, to $4.56 \mathrm{~W}$. This value is high because of other active peripherals on the board (which are not used by our SLAM application): Ethernet controller, LEDs, OLED display...

The performance measures (in frames per second) of the successive versions of our SLAM application along our co-design process are given in Table 5. The first column presents our SLAM task execution time with re-timing; the second, third and fourth columns present the impact on these performances of the progressive integration of 


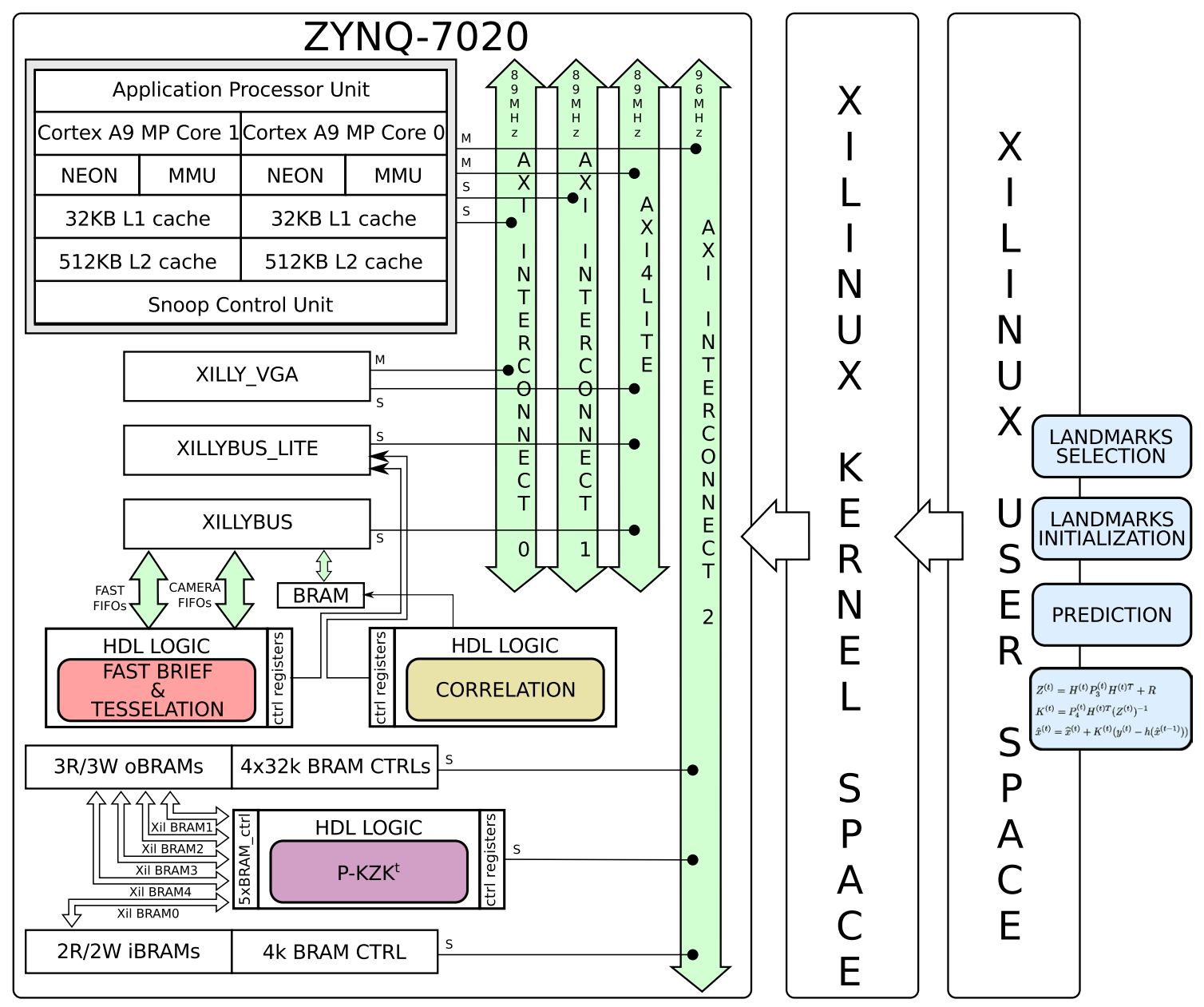

Fig. 23 Embedded SLAM's SoC architecture

Table 4 Global prototype's resource usage with IPs' performance

\begin{tabular}{lll}
\hline Resources & Instantiated & $\%$ FPGA \\
\hline LUTs & 27256 & 50 \\
Slice registers & 34127 & 31 \\
DSP48E & 40 & 18 \\
BRAM (36 kb) & 51 & 36 \\
BRAM $(18 \mathrm{~kb})$ & 15 & 6 \\
ARM Cortex A9 & 2 (cores) & 100 \\
$F_{\text {MAX }}(\mathrm{MHz})$ & Individually & Integrated \\
FAST+Tessellation & 166 & 89 \\
Correlator & 204 & 89 \\
Eq $P$ - KZK & 119 & 96 \\
Power consumption $(\mathrm{W})$ & & $4.56(0.671)$ \\
ZedBoard & - & \\
\hline
\end{tabular}

our hardware accelerators. The total execution time of the functional blocks is measured using software-intrusive code profiling. FIFO communication between the software threads takes a lot of time (12 ms). The loading of
Table 5 Execution time of SLAM functional blocks in (ms) on ZedBoard

\begin{tabular}{lllll}
\hline Iteration no. & I & II & III & IV \\
\hline COM & 12 & 12 & 12 & 12 \\
Landmark selection & 0.8 & 0.8 & 0.8 & 0.8 \\
Prediction & 2.1 & 2.1 & 2.1 & 2.1 \\
Correction_slam & 24 & 24 & 5 & 5 \\
Landmark initialization & 0.3 & 0.3 & 0.3 & 0.3 \\
Camera & 13 & 13 & 13 & 13 \\
Landmark correlation & 8.8 & 8.8 & 8.8 & 0.6 \\
Feature detection & 140 & 15 & 15 & 15 \\
FPS rate (Hz) & 5 & 16 & 20 & 24 \\
\hline
\end{tabular}

the frames from external memory also consumes a large part of the processing time $(13 \mathrm{~ms})$. Although the features detection task is much faster now, a huge percentage of the execution time is due to FIFO communication: $15-(640 \times 480) / 89 \mathrm{MHz}=11.55(\mathrm{~ms})$. The accelerated correction task takes only $20 \times 0.25=5(\mathrm{~ms})$, where $0.25 \mathrm{~ms}$ 


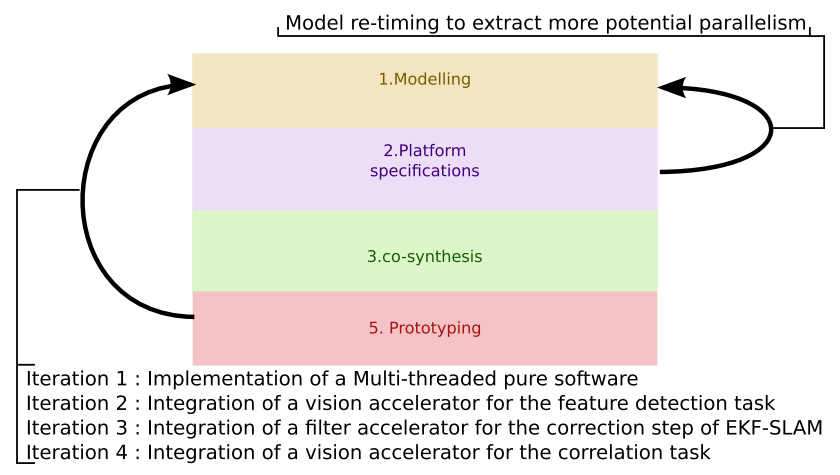

Fig. 24 Summary over the iterative prototyping

is the time needed for a single landmark correction. Thanks to multi-core correlation, matching of observed landmarks takes minimal time now.

Since the target-compliant prototype lacks real-time execution because of Xillinux communication interfaces, FIFO inter-thread communication and the latency needed to fetch images from a storage medium, we conclude our approach to be validated and our global prototype to meet the applicative constraints after four iterations, see Fig. 24.

\subsection{Comparison with the state of the art}

Three heterogeneous FPGA-based SLAM systems exist up to our knowledge: the monocular 2D EKF-SLAM of Bonato et al. [5], the C-SLAM monocular 3D EKF-SLAM previously implemented at the LAAS laboratory [20], and the 3D bundle adjustment SLAM of Nikolic et al. [32]. However, these works do not present complete SLAM systems as they do not implement the vision and pose estimation-related accelerators on a single reconfigurable device. The first paper does not mention what algorithm for feature detection the authors use, and moreover, their landmarks map is $2 \mathrm{D}$, which significantly downsizes the complexity of their problem compared to ours. On the other hand, the authors of the third paper use a ZynQ device for preprocessing vision tasks-feature detection and correlation, using multiple cameras along with synchronized IMU data. Moreover, they mention in conclusion that their future work envisages a "SLAM in a box" module on a single embedded device, as the computationally demanding bundle adjustment is ported onto a power-consuming Core2Duo host. Last, authors in [20] implement a monocular SLAM chain similar to ours; although they achieve performances similar to ours $(24 \mathrm{~Hz})$ with the same amount of observations and corrections, they consume more power (as they use two FPGAs). Compared to optimized software implementation like the one presented in Vincke et al. [45], our prototype achieves better frame rate at higher image resolution. One key advantage of our solution compared to software-only prototypes (even multi-core ones) is that the EKF part of the application is fully independent from the vision-processing operators. This allows our prototype to support higher image resolution with no additional CPU load.

\section{Conclusion and future works}

\subsection{Conclusion}

In this paper, we have implemented on a heterogeneous software/hardware architecture (Zynq FPGA) the first complete monocular 3D EKF-SLAM chain meeting the constraints of an ADAS (processing times, power consumption and design footprint). To do so, we have adapted and refined a classical co-design approach to encompass a hardware-in-theloop approach allowing to progressively integrate hardware accelerators; in our approach, the hardware accelerators are integrated one by one, based on the use of a systematic rule on the results of the cost profiling of the functional components of the heterogeneous SLAM chain under construction. The proposed co-design approach is generic and reusable for any advanced image processing chain on a heterogeneous architecture. Using a systematic rule paves the way towards some automation in the co-design approach proposed. We also have developed and validated several original hardware accelerators reusable for any embedded SLAM application or vision application involving the same functions. We have proposed and validated hardware IPs of all the image processing functions involved and of some data-processing functions involved in the numerical filtering steps. Our FAST hardware accelerator brings some progress with respect to the state of the art from the point of view of resources used and processing times. We think that our hardware accelerator for the computation of a BRIEF descriptor provides a good trade-off between resource consumption and performances. Our hardware accelerator for correlation implements 20 cores in parallel and also provides a hardware accelerator for the computation of a Hamming distance. The processing time obtained does not depend on the size of the correlation windows.

\subsection{Future work}

In our future works, we will interface the ZynQ-7020 device directly with an embedded camera (see Fig. 25), as we presumed that the camera task outputs a 1D pixel flow in our application model (data flow). That would remove the 13-ms Xillibus communication delay, which would yield an overperforming prototype.

Once the embedded camera has been wired with the ZynQ, our prototype may be evolved into a consumer-grade product. We intend to exploit the three developed harware 


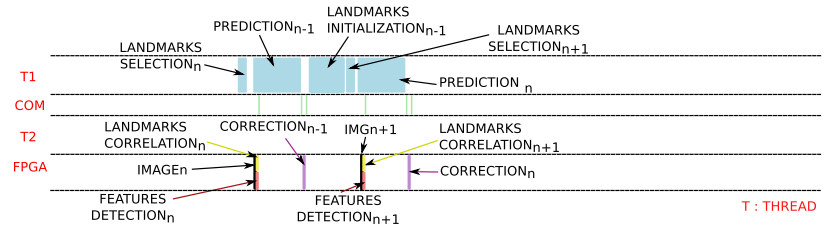

Fig. 25 Expected schedule of the SLAM application after fifth partitioning

IPs with the rescheduled EKF-SLAM application in a baremetal design where the reconfigurable fabric would permit a higher bandwidth (at least $150 \mathrm{MHz}$ instead of $89 \mathrm{MHz}$ ). On the other hand, once freed from the delays induced by the FIFO software communication and Xillinux interfaces, we estimate $^{1}$. that vision tasks and filtering tasks would roughly take at most $4.1 \mathrm{~ms}$ and $8.2 \mathrm{~ms}$, respectively, the filtering part being then the new bottleneck. That would roughly lead us to a monocular EKF-SLAM functionality with local maps containing 20 landmark points (while observing all of them) exceeding a $100-\mathrm{Hz}$ rate at very low power consumption, which would even outperform the RTSLAM's [37] execution (when using the same parameters as ours) on an Intel i7 core.

Acknowledgements This work is related to DICTA, a project funded by the "Midi-Pyrénées" region, and involving the LAAS-CNRS, the LGP-ENIT and the DTSO (Delta Technologies Sud-Ouest) company. F. Brenot's PhD work has been funded by the "Midi-Pyrénées" region and DTSO. This work has been co-authored by Daniel Tortei, whose $\mathrm{PhD}$ work has been funded by the FUI-AAP14 AIR-COBOT project, co-funded by BPI France, FEDER and the "Midi-Pyrénées" region.

\section{References}

1. Abrate, F., Bona, B., Indri, M.: Experimental EKF-based SLAM for mini-rovers with IR sensors only. In: EMCR (2007). http:// dblp.uni-trier.de/db/conf/emcr/emcr2007.html\#AbrateBI07. Accessed 14 Nov 2018

2. Bay, H., Ess, A., Tuytelaars, T., Van Gool, L.: Speeded-up robust features (SURF). Comput. Vis. Image Underst. 110(3), 346-359 (2008). https://doi.org/10.1016/j.cviu.2007.09.014

3. Bezati, E., Thavot, R., Roquier, G., Mattavelli, M.: High-level dataflow design of signal processing systems for reconfigurable and multicore heterogeneous platforms. J. Real Time Image Proc. 9(1), 251-262 (2014). https://doi.org/10.1007/s11554-013-0326-5

4. Birem, M., Berry, F.: FPGA-based real time extraction of visual features. In: 2012 IEEE International Symposium on Circuits and Systems, pp. 3053-3056. (2012). https://doi.org/10.1109/ISCAS .2012 .6271964

5. Bonato, V., Marques, E., Constantinides, G.A.: A floating-point extended Kalman filter implementation for autonomous mobile robots. J. VLSI Sig. Proc. (2008). https://doi.org/10.1109/ FPL.2007.4380720

6. Botero, D., Gonzalez, A., Devy, M., et al.: Architecture embarquée pour le SLAM monoculaire. In: Proceedings of RFIA 2012 (2012)

1 This estimation is made upon the timings presented in Table 5.
7. Brenot, F., Fillatreau, P., Piat, J.: FPGA based accelerator for visual features detection. In: International Workshop IEEE Electronics, Control, Measurement, Signals and their application to Mechatronics (ECMSM), Liberec, Czech Republic (2015). https ://hal.archives-ouvertes.fr/hal-01300912. Accessed 14 Nov 2018

8. Calonder, M., Lepetit, V., Strecha, C., Fua, P.: BRIEF: Binary Robust Independent Elementary Features. In: Daniilidis, K., Maragos, P., Paragios, N. (eds.) Computer Vision-ECCV 2010, vol. 6314. Springer, Berlin, Heidelberg (2010)

9. Calvez, J.P., Pasquier, O., Heller, D.: Hardware/Software System Design Based on the MCSE Methodology, pp. 115-150. Springer, Boston (1997). https://doi.org/10.1007/978-1-4615-6295-56

10. Chatila, R., Laumond, J.: Position referencing and consistent world modeling for mobile robots. In: Proceedings. 1985 IEEE International Conference on Robotics and Automation, vol. 2, pp. 138-145 (1985). https://doi.org/10.1109/ROBOT.1985.1087373

11. Civera, J., Grasa, O.G., Davison, A.J., Montiel, J.M.M.: 1-point RANSAC for EKF-based structure from motion. In: Intelligent Robots and Systems, 2009. IROS 2009. IEEE/RSJ International Conference, pp. 3498-3504 (2009). https://doi.org/10.1109/ IROS.2009.5354410

12. Davison, A.J., Reid, I.D., Molton, N.D., Stasse, O.: MonoSLAM: real-time single camera SLAM. IEEE Trans. Pattern Anal. Mach. Intell. 29(6), 1052-1067 (2007). https://doi.org/10.1109/TPAMI .2007 .1049

13. Devy, M., Boizard, J.L., Galeano, D.B., Lindado, H.C., Manzano, M.I., Irki, Z., Naoulou, A., Lacroix, P., Fillatreau, P., Fourniols, J.Y., Parra, C.: Chapter 17. stereovision algorithm to be executed at $100 \mathrm{hz}$ on a FPGA-based architecture. In: Bhatti, A. (ed.) Advances in Theory and Applications of Stereo Vision, pp. 327-352. InTech (2011). http://oatao.univ-toulouse.fr/12030/, thanks to Intech's Publishing. The definitive version is available at http://www.intechopen.com/books/advances-in-theory-and-appli cations-of-stereo-vision/stereovision-algorithm-to-be-executed-at100hz-on-a-fpga-based-architecture. Accessed 14 Nov 2018

14. Durrant-Whyte, H., Bailey, T.: Simultaneous localization and mapping: part I. IEEE Robot. Autom. Mag. 13(2), 99-110 (2006). https://doi.org/10.1109/MRA.2006.1638022

15. Faessler, M., Fontana, F., Forster, C., Mueggler, E., Pizzoli, M., Scaramuzza, D.: Autonomous, vision-based flight and live dense $3 \mathrm{~d}$ mapping with a quadrotor micro aerial vehicle. J. Field Robot. 33(4), 431-450 (2015)

16. Fisher, R.B., Breckon, T.P., Dawson-Howe, K., Fitzgibbon, A., Robertson, C., Trucco, E., Williams, C.K.I.: Dictionary of Computer Vision and Image Processing, 2nd edn. Wiley Publishing, Hoboken (2013)

17. Fowers, J., Brown, G., Cooke, P., Stitt, G.: A performance and energy comparison of FPGAS, GPUS, and multicores for sliding-window applications. In: Proceedings of the ACM/SIGDA International Symposium on Field Programmable Gate Arrays. ACM, New York, USA, FPGA '12, pp. 47-56 (2012). https://doi. org/10.1145/2145694.2145704

18. Franke, U., Gavrila, D., Gern, A., Görzig, S., Janssen, R., Paetzold, F., Wöhler, C.: 6-From door to door-principles and applications of computer vision for driver assistant systems. In: Vlacic, L., Harashima, F., Parent, M. (eds.) Intelligent vehicles technologies: theory and applications, pp. 131-188. Butterworth Heinemann, Oxford (2001). https://doi.org/10.1016/B978-0750650939/50008-6

19. Gohringer, D., Birk, M., Dasse-Tiyo, Y., Ruiter, N., Hübner, M., Becker, J.: Reconfigurable MPSoC versus GPU: performance, power and energy evaluation. In: Proceedings of IEEE International Conference on Industrial Informatics (INDIN), Lisbon (2011). https://doi.org/10.1109/INDIN.2011.6035003

20. Gonzalez, A., Codol, J.M., Devy, M.: A C-embedded algorithm for real-time monocular SLAM. In: 18th International 
Conference on Electronics, Circuits and Systems, Beyrouth, Liban (2011). https://doi.org/10.1109/ICECS.2011.6122362

21. Harris, C., Stephens, M.: A combined corner and edge detector. In: Proceedings of Fourth Alvey Vision Conference, pp. 147-151 (1988)

22. Jovanovic, Z., Milutinovic, V.: FPGA accelerator for floatingpoint matrix multiplication. IET Comput. Digit. Tech. (2012). https://doi.org/10.1049/iet-cdt.2011.0132

23. Kienhuis, B., Deprettere, E., Vissers, K., Van Der Wolf P.: An approach for quantitative analysis of application-specific dataflow architectures. In: Proceedings IEEE International Conference on Application-Specific Systems, Architectures and Processors, pp. 338-349 (1997). https://doi.org/10.1109/ ASAP.1997.606839

24. Kraft, M., Schmidt, A., Kasinski, A.J.: High-speed image feature detection using FPGA implementation of fast algorithm. In: Proceedings of the International Conference on Computer Vision Theory and Applications (2008)

25. Kukkala, P., Riihimaki, J., Hannikainen, M., Hamalainen, T.D., Kronlof, K.: UML 2.0 profile for embedded system design. In: Design, Automation and Test in Europe, vol. 2, pp. 710-715 (2005). https://doi.org/10.1109/DATE.2005.321

26. de Lima, R., Martinez-Carranza, J., Morales-Reyes, A., Cumplido, R.: Accelerating the construction of brief descriptors using an FPGA-based architecture. In: 2015 International Conference on ReConFigurable Computing and FPGAs (ReConFig), pp. 1-6 (2015). https://doi.org/10.1109/ReCon Fig.2015.7393285

27. Loose, H., Franke, U.: B-spline-based road model for $3 \mathrm{~d}$ lane recognition. In: 13th International IEEE Conference on Intelligent Transportation Systems, pp. 91-98 (2010). https://doi. org/10.1109/ITSC.2010.5624968

28. Lowe, D.G.: Object recognition from local scale-invariant features. In: Computer Vision, 1999. The Proceedings of the Seventh IEEE International Conference, vol. 2, pp. 1150-1157 (1999). https://doi.org/10.1109/ICCV.1999.790410

29. Montemerlo, M., Thrun, S., Koller, D., Wegbreit, B.: FastSLAM: a factored solution to the simultaneous localization and mapping problem. In: Proceedings of the AAAI National Conference on Artificial Intelligence. AAAI, Edmonton, Canada (2002)

30. Mouragnon, E., Dekeyser, F., Sayd, P., Lhuillier, M., Dhome, M.: Real time localization and 3D reconstruction. In: Proceedings of the IEEE computer society conference on computer vision and pattern recognition, vol. 1, pp. 363-370 (2006)

31. Moutarlier, P., Chatila, R.: An Experimental System for Incremental Environment Modelling by an Autonomous Mobile Robot. In: Hayward, V., Khatib, O. (eds.) Experimental Robotics, pp. 327-346. Springer, Berlin (1990). https://doi.org/10.1007/BFb00 42528

32. Nikolic, J., Rehder, J., Burri, M., Gohl, P., Leutenegger, S., Furgale, P.T., Siegwart, R.: A synchronized visual-inertial sensor system with FPGA pre-processing for accurate real-time SLAM. In: IEEE International Conference on Robotics and Automation (ICRA) (2014)

33. Okuda, R., Kajiwara, Y., Terashima, K.: A survey of technical trend of ADAS and autonomous driving. In: Proceedings of Technical Program-2014 International Symposium on VLSI Technology, Systems and Application (VLSI-TSA), pp. 1-4 (2014). https ://doi.org/10.1109/VLSI-TSA.2014.6839646

34. Pelcat, M., Piat, J., Wipliez, M., Aridhi, S., Nezan, J.F.: An open framework for rapid prototyping of signal processing applications. EURASIP J. Embed. Syst. 2009(1), 598529 (2009). https://doi. org/10.1155/2009/598529

35. Petersen, R.J., Hutchings, B.: An assessment of the suitability of FPGA-based systems for use in digital signal processing. In:
Proceedings of the 5th International Workshop on Field-Programmable Logic and Applications, pp. 293-302. Springer, London, FPL '95 (1995). http://dl.acm.org/citation.cfm?id=647922.74088 3. Accessed 14 Nov 2018

36. Rosten, E., Drummond, T.: Fusing points and lines for high performance tracking. In: Tenth IEEE International Conference on Computer Vision (ICCV'05) Volume 1, vol. 2, pp. 1508-1515 (2005). https://doi.org/10.1109/ICCV.2005.104

37. Roussillon, C., Gonzalez, A., Solà, J., Codol, J., Mansard, N., Lacroix, S., Devy, M.: RT-SLAM : a generic and real-time visual SLAM implementation. In: 8th International Conference on Computer Vision Systems, Sophia Antipolis (France) (2011)

38. Shaout, A., El-mousa, A.H., Mattar, K.: Specification and modeling of HW/SW co-design for heterogeneous embedded systems. In: Proceedings of the World Congress on Engineering, vol. 1 (2009)

39. Shi, J., Tomasi, C.: Good features to track. In: 1994 Proceedings of IEEE Conference on Computer Vision and Pattern Recognition, pp. 593-600 (1994) https://doi.org/10.1109/CVPR.1994.323794

40. Solà, J.: Towards visual localization, mapping and moving objects tracking by a mobile robot: a geometric and probabilistic approach. Theses, Institut National Polytechnique de Toulouse-INPT (2007). https://tel.archives-ouvertes.fr/tel-00136 307. Accessed 14 Nov 2018

41. Sérot, J., Berry, F., Bourrasset, C.: High-level dataflow programming for real-time image processing on smart cameras. J. Real Time Image Proc. (2014). https://doi.org/10.1007/s1155 4-014-0462-6

42. Strasdat, H., Montiel, J.M.M., Davison, A.J.: Real-time monocular SLAM: why filter? In: Proceedings of IEEE International Conference on Robotics and Automation (ICRA) (2010). https://doi. org/10.1109/TPAMI.2007.1049

43. Tertei, D.T., Piat, J., Devy, M.: FPGA design and implementation of a matrix multiplier based accelerator for 3D EKF SLAM. In: Proceedings of European Conference on ReConFigurable Computing and FPGAs (ReConFig) (2014)

44. Thrun, S., Burgard, W., Fox, D.: Probabilistic Robotics. MIT Press, Cambridge (2005). https://doi.org/10.1162/artl.2008.14.2.227

45. Vincke, B., Elouardi, A., Lambert, A.: Real time simultaneous localization and mapping: towards low-cost multiprocessor embedded systems. EURASIP J. Embed. Syst. (2012). https://doi. org/10.1186/1687-3963-2012-5

46. Vincke, B., Elouardi, A., Lambert, A., Merigot, A.: Efficient implementation of SLAM on a multi-core embedded system. In: IECON 2012 - 38th Annual Conference on IEEE Industrial Electronics Society, pp. 3049-3054 (2012). https://doi.org/10.1109/ IECON.2012.6389411

47. Xillinux.: Xillinux: A Linux distribution for Zedboard, ZyBo, MicroZed and SocKit (2010). http://xillybus.com/xillinux. Accessed 14 Nov 2018

48. Yao, L., Feng, H., Zhu, Y., Jiang, Z., Zhao, D., Feng, W.: An architecture of optimised SIFT feature detection for an FPGA implementation of an image matcher. In: Field-Programmable Technology, 2009. FPT 2009. International Conference, pp. 30-37 (2009). https://doi.org/10.1109/FPT.2009.5377651

49. Yviquel, H., Lorence, A., Jerbi, K., Cocherel, G., Sanchez, A., Raulet, M.: ORCC: multimedia development made easy. In: Proceedings of the 21st ACM International Conference on Multimedia. ACM, MM '13, pp. 863-866 (2013). https://doi. org/10.1145/2502081.2502231

50. Zhuo, L., Prasanna, V.K.: Scalable and modular algorithms for floating-point matrix multiplication on reconfigurable computing systems. In: IEEE Transactions on Parallel and Distributed Systems, vol. 18, No. 4 (2007). https://doi.org/10.1109/ TPDS.2007.1001 
Jonathan Piat is an associate professor at the Department of Electrical and Computer Engineering at Paul Sabatier University of Toulouse (France). He holds a joint appointment in the Laboratory for Analysis and Architecture of Systems, a CNRS research unit. Dr. Piat obtained his $\mathrm{PhD}$ thesis in electronic and signal processing from the INSA of Rennes in 2010. His work deals with the integration of robotics algorithms on FPGA-based dedicated hardware. His main research interests include data flow models, hardware/software co-design, embedded vision processing and robotics.

Philippe Fillatreau graduated from the ENSEEIHT engineering school in 1988. He obtained his $\mathrm{PhD}$ in perception for mobile robotics in 1994 at the LAAS-CNRS laboratory, Toulouse, France. His PhD works, funded by the Framatome (now Areva) company, dealt with perception, 3D environment modelling and localisation for an autonomous all-terrain mobile robot, in the framework of joint projects dealing with public safety or planetary exploration. In 1991-1992, he spent 1 year at the Matra Marconi Space (now Airbus Group Defence and Space) company, where he was involved in the Eureka-AMR project. In 1994-1995, he was a post-doctoral visiting researcher at the Department of Artificial Intelligence of the University of Edinburgh, Scotland; funded by a Human Capital and Mobility fellowship of the European Community, his works dealt with non-polyhedral object recognition and model update for public safety mobile robots. In 1996, Philippe Fillatreau joined the Delta Technologies Sud-Ouest company (Toulouse) where he was technical head of the computer vision activities. There, he led the research and industrial projects in the fields of Computer
Vision and Algorithm Architecture Adequacy, aiming notably at integrating advanced image processing algorithms in smart cameras based on FPGAs for very high-throughput applications (e.g. transports safety, online satellite images processing or quality control of industrial processes). In 2009, he joined the Ecole Nationale d'Ingénieurs de Tarbes as a researcher and lecturer. His current research interests are related to virtual reality, computer vision, mobile robotics and hardware/software co-design of embedded vision systems.

Michel Devy is an engineer from ENSIMAG (1976, Grenoble, France), doctorate in Computer Science (1980, Toulouse, France), and CNRS Director of Research at Laboratory of Analysis and Architecture of Systems (LAAS-CNRS) (2000, Toulouse). For more than 35 years, he has contributed to the Robotics Department, now member (as team leader until 2013) of the research group RAP (Robotics, Action and Perception). His research has been devoted to the application of computer vision in automation and robotics. He was involved in numerous national and international projects, about manufacturing, mobile robots for space exploration and for civil safety, 3D modeling, intelligent vehicles, service robotics, etc. His current research interests concern at first perception for mobile robots navigating in natural or indoor environments, e.g. visual-based navigation and manipulation, 3D object modelling, Simultaneous Localization and Mapping (SLAM), design and implementation of integrated sensors, etc. He was $\mathrm{PhD}$ advisor or co-advisor for about $45 \mathrm{PhD}$ students and co-author of about 350 scientific communications. He was responsible for the Technical Committee, Intelligent and Autonomous Vehicles at IFAC from 2008 to 2011. 